\title{
The Development of Severe Vortices within Simulated High-Shear, Low-CAPE Convection
}

\author{
KeIth D. SHERburn ${ }^{\mathrm{a}}$ AND MATTHEW D. PARKER \\ Department of Marine, Earth, and Atmospheric Sciences, North Carolina State University, Raleigh, North Carolina
}

(Manuscript received 15 July 2018, in final form 23 March 2019)

\begin{abstract}
Environments characterized by large values of vertical wind shear and modest convective available potential energy (CAPE) are colloquially referred to as high-shear, low-CAPE (HSLC) environments. Convection within these environments represents a considerable operational forecasting challenge. Generally, it has been determined that large low-level wind shear and steep low-level lapse rates-along with synoptic-scale forcing for ascent-are common ingredients supporting severe HSLC convection. This work studies the specific processes that lead to the development of strong surface vortices in HSLC convection, particularly associated with supercells embedded within a quasi-linear convective system (QLCS), and how these processes are affected by varying low-level shear vector magnitudes and lapse rates. Analysis of a control simulation, conducted with a base state similar to a typical HSLC severe environment, reveals that the key factors in the development of a strong surface vortex in HSLC embedded supercells are (i) a strong low- to midlevel mesocyclone, and (ii) a subsequent strong low-level updraft that results from the intense, upward-pointing dynamic perturbation pressure gradient acceleration. Through a matrix of high-resolution, idealized simulations, it is determined that sufficient low-level shear vector magnitudes are necessary for the development of low- to midlevel vertical vorticity [factor (i)], while steeper low-level lapse rates provide stronger initial low-level updrafts [factor (ii)]. This work shows why increased low-level lapse rates and low-level shear vector magnitudes are important to HSLC convection on the storm scale, while also revealing similarities between surface vortexgenesis in HSLC embedded supercells and higherCAPE supercells.
\end{abstract}

\section{Introduction}

Schneider et al. (2006) first referred to severe convection occurring within environments characterized by strong vertical wind shear, modest convective available potential energy (CAPE), and plentiful low-level moisture as one of the two "key subclasses" of severe weather in the United States. These high-shear, low-CAPE (HSLC) environments support approximately half of the significant $(\mathrm{EF} 2+)$ tornadoes in the CONUS (Schneider et al. 2006), either within miniature supercells or quasi-linear convective systems (QLCSs; e.g., Smith et al. 2012; Davis and Parker 2014). However, HSLC convection is also associated with low probabilities of detection (PODs) and high false alarm rates (FARs) of tornado watch and warning

\footnotetext{
${ }^{a}$ Current affiliation: National Weather Service Forecast Office, Rapid City, South Dakota.
}

Corresponding author: Keith Sherburn, keith.sherburn@ noaa.gov products (Dean and Schneider 2012; Anderson-Frey et al. 2016; Sherburn et al. 2016).

Recent work has improved our knowledge of the ingredients and settings favoring severe HSLC convection (Sherburn and Parker 2014; Sherburn et al. 2016; King et al. 2017). Severe HSLC environments tend to exhibit steeper low-level lapse rates, larger low-level shear vector magnitudes, and stronger synoptic-scale forcing for ascent than their nonsevere counterparts. However, due to very few targeted observations or high-resolution numerical simulations of HSLC events, there remain many gaps in our knowledge regarding the dynamics that govern the differences between severe and nonsevere HSLC convection. The primary aim of the following idealized simulations is to understand the links in the chain that lead from the development of a strong updraft to the subsequent genesis of a strong surface (i.e., lowest model level) vortex in a typical HSLC severe convection environment. Once an understanding of these processes is established, it will be possible to assess what links in this chain are "broken" in some portions of the parameter space. 


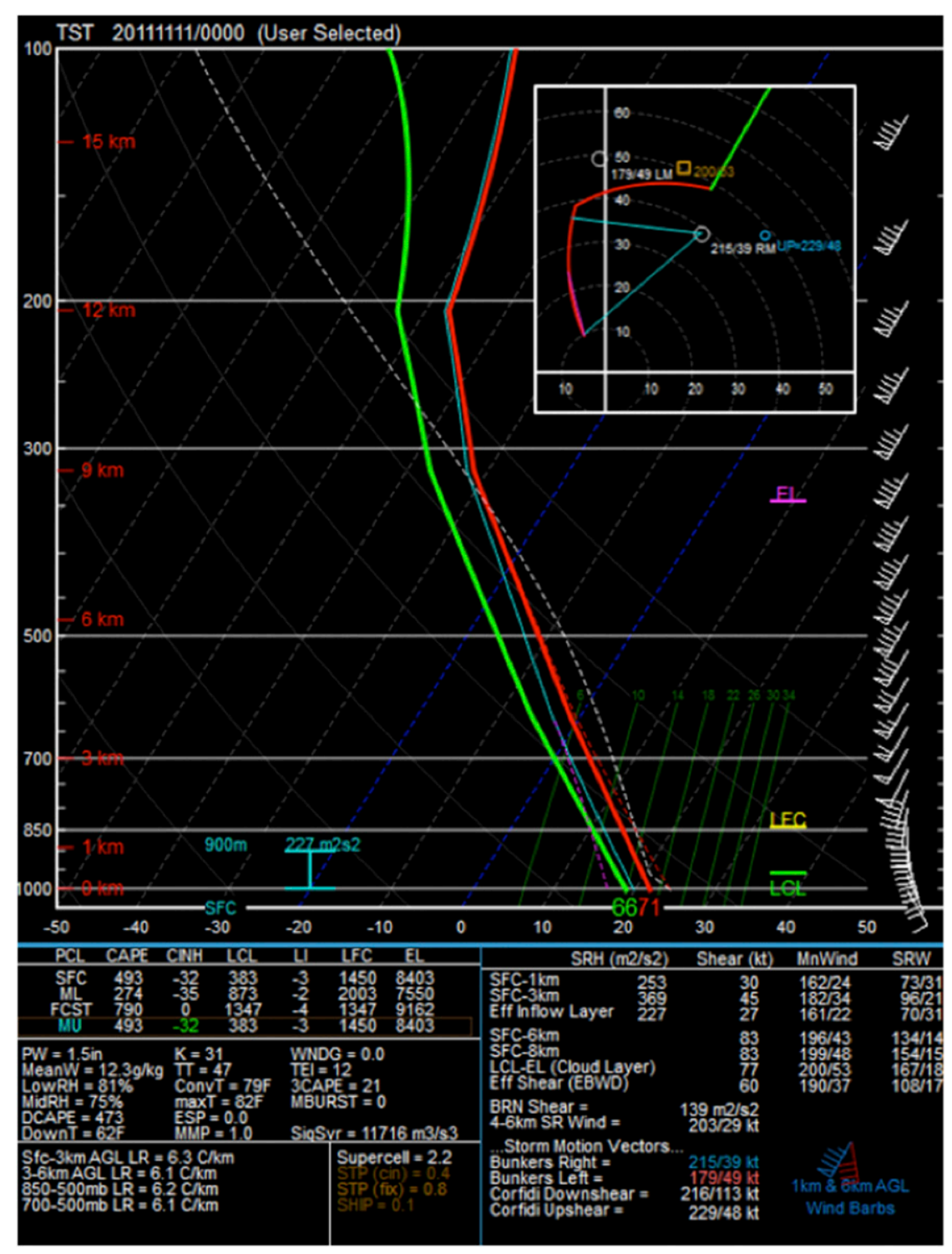

FIG. 1. Control base-state environment in HSLC matrix of simulations.

For the most part, research on the genesis of nearsurface vortices and tornadoes has been focused on high-CAPE environments supportive of supercells, which are common in the U.S. Great Plains. Tornadoes develop within high-CAPE supercells following the development of a midlevel mesocyclone-the vorticity of which is acquired from the tilting of environmental horizontal vorticity, present due to sufficient vertical wind shear-and the development of a separate area of vertical vorticity $(\zeta)$ near the surface. Subsequently, this near-surface $\zeta$ is converged and stretched, producing a tornado (Davies-Jones and Brooks 1993; Davies-Jones 2015). The first step in this process is fairly well established (e.g., Klemp 1987). However, pathways for the development and intensification of surface $\zeta$ remain a subject of much investigation (e.g., Dawson et al. 2016; Markowski and Richardson 2017; Roberts et al. 2016; Coffer and Parker 2017; Rotunno et al. 2017).

Recent work has elucidated the mechanisms by which surface $\zeta$ arises within supercells and how it is intensified to tornadic strength (e.g., Markowski and Richardson 2014; Dahl et al. 2014; Roberts et al. 2016). Via trajectory analysis, these studies have also identified the general pathways by which parcels contribute to intense near-surface vortices. In most studies, the majority of parcels contributing to near-surface vortices arrive from storm-relative north or northwest, acquiring vertical vorticity as they descend in a downdraft 
(e.g., Dahl et al. 2014). When surface drag is included, it has been shown to play a large role in the production of vorticity that is subsequently tilted and stretched into a tornado-like vortex (e.g., Schenkman et al. 2012, 2014; Roberts et al. 2016), although the most physically consistent way to include surface drag in an idealized model remains uncertain (e.g., Markowski and Bryan 2016; Markowski 2018). More recent numerical simulations with lowest model levels very close to the ground (e.g., Rotunno et al. 2017) suggest that $\zeta$ may arise due to tilting of baroclinically generated horizontal vorticity very close to the surface. Practically speaking, the source of surface $\zeta$ may not be the main concern for operational meteorologists; rather, the ability for the storm's updraft to converge and stretch this vorticity is the deciding factor in whether or not it produces a tornado (e.g., Coffer et al. 2017). Therefore, the strength of low-level vertical accelerations-and subsequently, low-level updrafts-is critical in distinguishing tornadic from nontornadic supercells.

In contrast to supercells, mesovortices within QLCSs are shallow and lack accompanying midlevel rotation (e.g., Trapp and Weisman 2003; Weisman and Trapp 2003; Wakimoto et al. 2006; Atkins and St. Laurent 2009a,b; Xu et al. 2015). Mesovortices that produce tornadoes or damaging straight-line winds tend to be longer lived, taller, and stronger than nonsevere mesovortices (e.g., Przybylinski et al. 2000; Atkins et al. 2004, 2005), characteristics that may depend upon the lowlevel shear vector magnitude (Weisman and Trapp 2003; Godfrey et al. 2004; Schaumann and Przybylinski 2012). Large values of low- to midlevel shear vector magnitude in HSLC environments may be associated with the large fraction of QLCS tornadoes within HSLC convection (Smith et al. 2012; Davis and Parker 2014) because they support deeper, longer-lived mesovortices.

Based on this prior research, the strength of low-level updrafts and associated rotation in mesocyclones or mesovortices influence the likelihood of tornadogenesis. It is important to consider the means by which environmental variables with documented discriminatory skill in HSLC environments (low-level lapse rates and shear vector magnitudes ${ }^{1}$; Sherburn and Parker 2014; Sherburn et al. 2016) could impact each of these

\footnotetext{
${ }^{1}$ Despite being a critical discriminator between severe and nonsevere HSLC convective events, the magnitude of synopticscale forcing is not systematically tested in this modeling study. The idealized framework of the experimental design does attempt to mimic synoptic-scale forcing, but it is not explicitly represented. As such, sensitivity studies associated with synoptic forcing cannot be performed within this framework and will be left for future work.
}

TABLE 1. Selected base-state environment variables for matrix of simulations.

\begin{tabular}{lcrrrr}
\hline \multicolumn{1}{c}{ Variable } & Control & $+01 \mathrm{~s}$ & $-01 \mathrm{~s}$ & $+031 \mathrm{r}$ & $-03 \mathrm{lr}$ \\
\hline SBCAPE $\left(\mathrm{J} \mathrm{kg}^{-1}\right)$ & 493 & 493 & 493 & 493 & 493 \\
MLCAPE $\left(\mathrm{J} \mathrm{kg}^{-1}\right)$ & 274 & 274 & 274 & 276 & 288 \\
0-3-km CAPE $\left(\mathrm{J} \mathrm{kg}^{-1}\right)$ & 21 & 21 & 21 & 40 & 6 \\
0-1-km shear $(\mathrm{kt})$ & 30 & 40 & 20 & 30 & 30 \\
0-1-km SRH $\left(\mathrm{m}^{2} \mathrm{~s}^{-2}\right)$ & 253 & 352 & 160 & 236 & 264 \\
0-3-km shear $\left(\mathrm{kt}^{2}\right.$ & 45 & 45 & 45 & 45 & 45 \\
0-3-km SRH $\left(\mathrm{m}^{2} \mathrm{~s}^{-2}\right)$ & 369 & 451 & 304 & 366 & 370 \\
0-6-km shear $(\mathrm{kt})$ & 83 & 83 & 83 & 83 & 83 \\
\hline
\end{tabular}

features. The sensitivity of vortexgenesis to low-level shear vector magnitude has been documented in both QLCSs and supercells within high-CAPE environments, as reviewed above. The strength and lifetime of QLCS mesovortices-and, accordingly, their potential to produce severe hazards-appears to primarily be dictated by the low-level environmental shear vector magnitude (Weisman and Trapp 2003; Schaumann and Przybylinski 2012). Meanwhile, shear vector magnitude and storm-relative helicity over the lowest 500 to $1000 \mathrm{~m}$ above ground has been shown to be a key discriminator between tornadic and nontornadic supercells (Brooks et al. 2003; Adlerman and Droegemeier 2005; Esterheld and Giuliano 2008; Markowski and Richardson 2014; Coffer and Parker 2017). Vortex sensitivity to low-level lapse rates is less definitive, though highly idealized simulations suggest that steeper lapse rates facilitate stronger vortices (Leslie and Smith 1978; Parker 2012). Before we can better prepare for severe potential within HSLC environments, we must understand the sensitivity of convection therein to these parameters with documented discriminatory skill. The specific question guiding this research is: by what mechanisms do changes in low-level lapse rates and shear vector magnitudes influence the evolution and severity of HSLC storms and their potential to support strong surface vortices? ${ }^{2}$

\section{Methodology}

\section{a. Model setup}

To address the question of how varying low-level lapse rates or shear vector magnitudes impacts HSLC convection, a multimember model sensitivity matrix was developed to examine how variations in $0-1-\mathrm{km}$ shear vector magnitude (01s) and $0-3-\mathrm{km}$ lapse rates

\footnotetext{
${ }^{2}$ We acknowledge that these sensitivities may vary depending on the convective mode.
} 


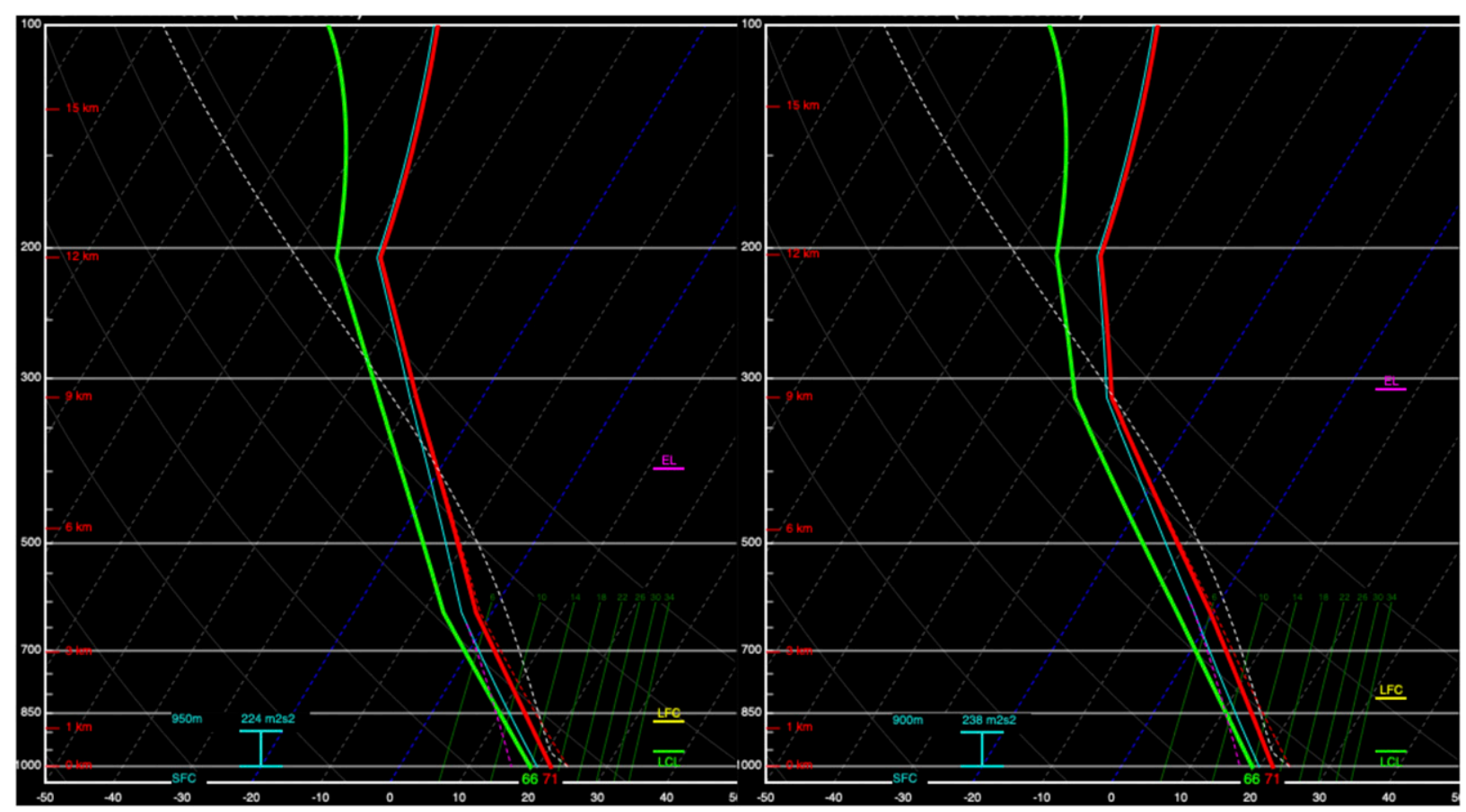

FIG. 2. Base-state thermodynamic profiles for the (left) increased low-level lapse rate and (right) decreased low-level lapse rate simulations.

(031r) affect the structure, evolution, and intensity of HSLC convection. The control thermodynamic and kinematic profiles were subjectively designed to capture the typical characteristics from prior HSLC composites (Sherburn et al. 2016) and preliminary radiosonde data from the Verifications of the Origins of Rotation in
Tornadoes Experiment-Southeast (VORTEX-SE) field experiment and HSLC-focused radiosonde launches from North Carolina State University (Sherburn et al. 2019). The control base-state environment (Fig. 1) exhibited $493 \mathrm{~J} \mathrm{~kg}^{-1}$ of surface-based (SB) CAPE, $21 \mathrm{~J} \mathrm{~kg}^{-1}$ of $0-3-\mathrm{km}$ CAPE, and 30,45 , and $83 \mathrm{kt}\left(1 \mathrm{kt} \approx 0.5144 \mathrm{~m} \mathrm{~s}^{-1}\right)$

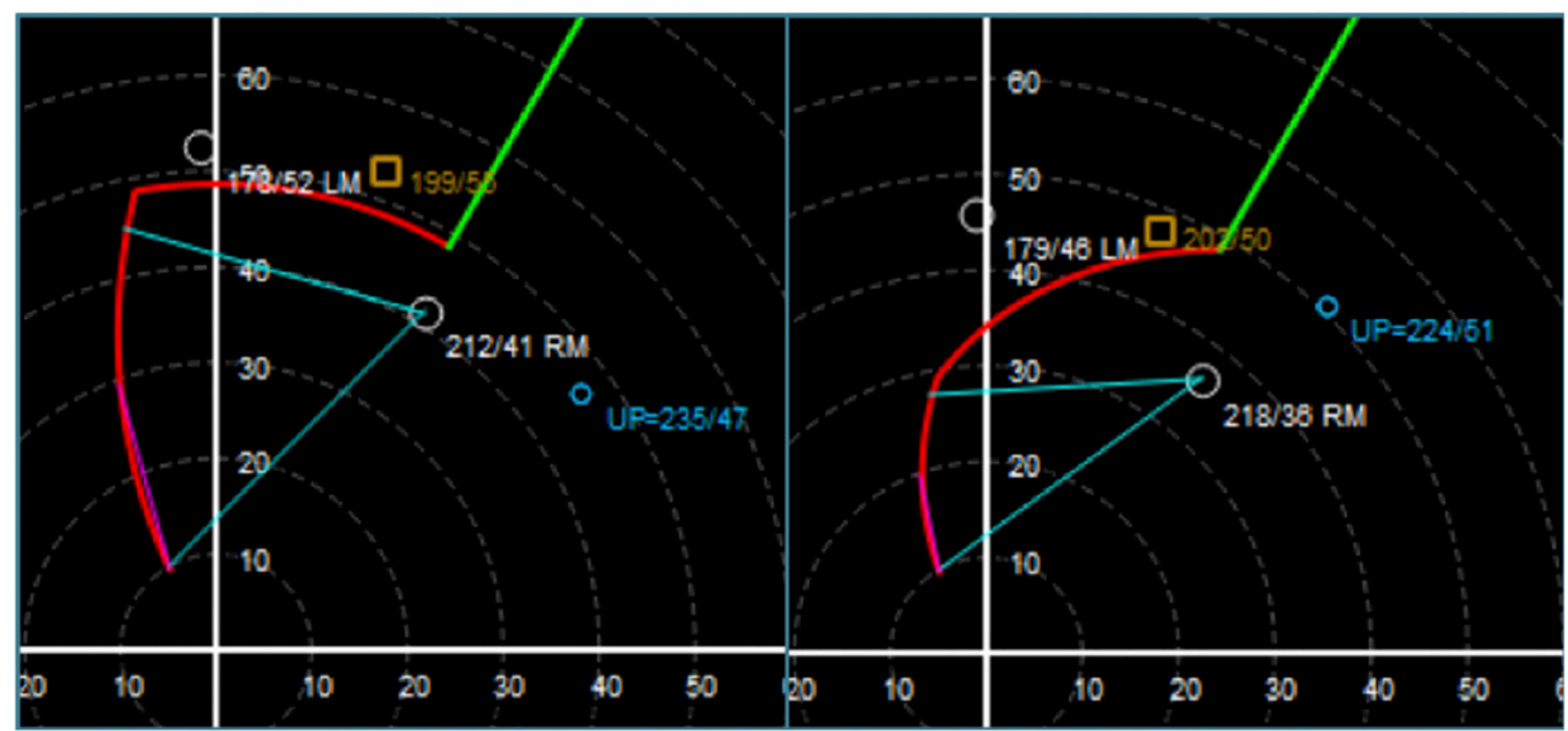

FIG. 3. Base-state kinematic profiles for the (left) increased low-level shear and (right) decreased low-level shear simulations. 

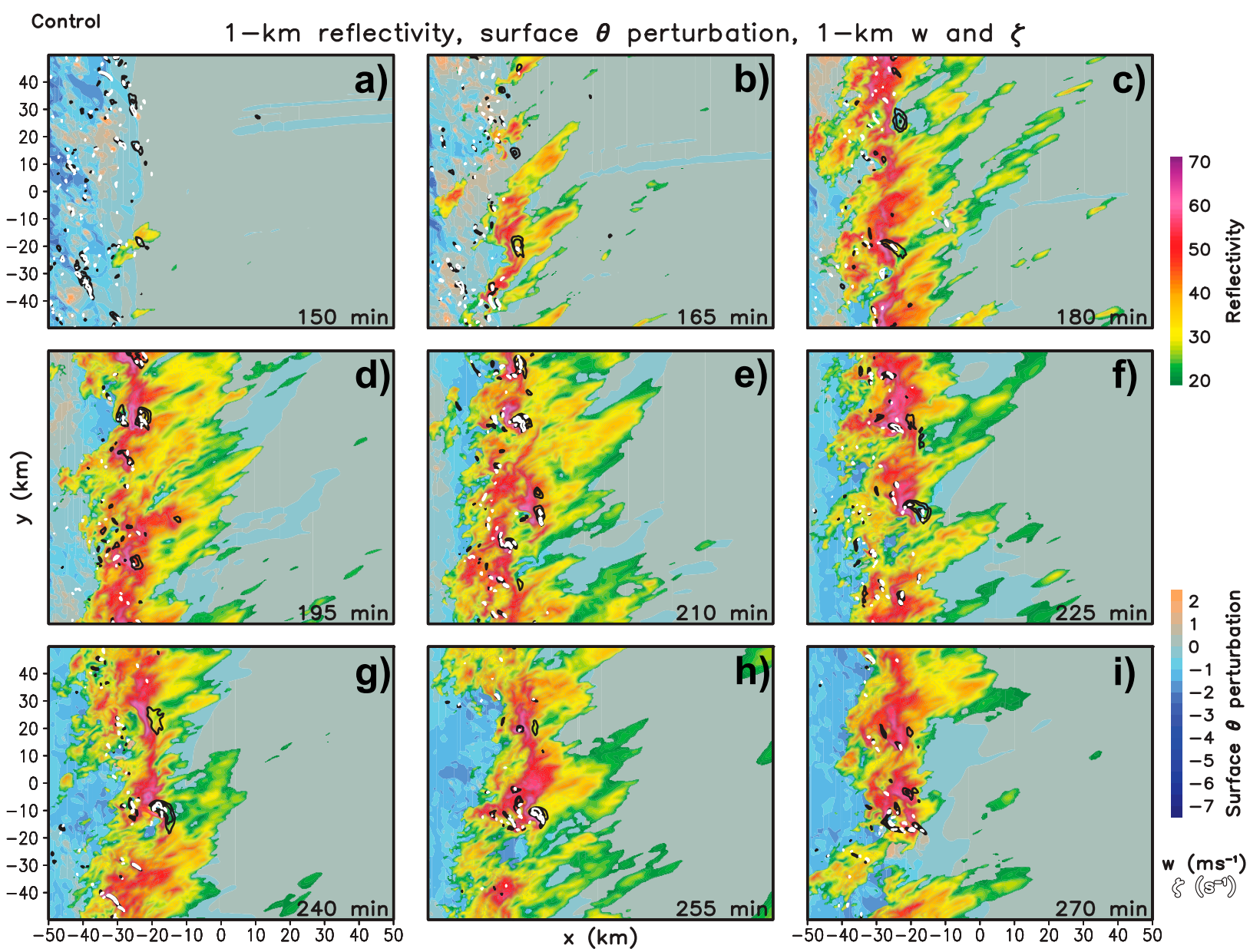

FIG. 4. Overview of 1-km reflectivity (dBZ; rainbow shading), surface potential temperature perturbation (K; tan to dark blue shading), $1-\mathrm{km} w\left(5,7.5,10,12.5,15,20,25\right.$, and $30 \mathrm{~m} \mathrm{~s}^{-1}$; black contours $)$, and $1-\mathrm{km} \zeta\left(0.02,0.04,0.06,0.08\right.$, and $0.1 \mathrm{~s}^{-1}$; white contours) for the control simulation from (a) $t=150 \mathrm{~min}$ through (i) $t=270 \mathrm{~min}$ over 15 -min intervals.

of $0-1-, 0-3-$, and $0-6-\mathrm{km}$ shear vector magnitude, respectively.

The four primary experimental simulations are characterized by adjustments to the base-state $01 \mathrm{~s}$ or $031 \mathrm{r}$ over ranges of $20(-01 \mathrm{~s})$ to $40 \mathrm{kt}(+01 \mathrm{~s})$ and $6.0(-031 \mathrm{r})$ to $6.5 \mathrm{~K} \mathrm{~km}^{-1}(+031 \mathrm{r}),{ }^{3}$ respectively. Table 1 shows the variability in chosen convective ingredients from the control base-state environment to the other simulations.

\footnotetext{
${ }^{3}$ This range of lapse rates seems fairly modest; however, climatologically, the inner $50 \%$ of $031 \mathrm{r}$ associated with severe HSLC convection for any given region tends to encompass a range of only about $1 \mathrm{~K} \mathrm{~km}^{-1}$ (Sherburn and Parker 2014). Using SPC mesoanalysis data for the Southeast United States, 25th and 75th percentile values are around $5-6 \mathrm{~K} \mathrm{~km}^{-1}$, respectively. Values on the higher side of this distribution were chosen for this particular study because lower values did not support appreciable convection in this idealized, homogeneous setup. Additionally, despite a fairly narrow range of lapse rates, the range of $0-3-\mathrm{km}$ CAPE values is rather large (Table 1).
}

Skew $T-\log p$ diagrams for the $031 \mathrm{r}$ variations are provided in Fig. 2, while Fig. 3 shows hodographs for the 01s variations. Storm-relative helicity (SRH) values change considerably with variations in $01 \mathrm{~s}$, as expected, with minor changes between the $031 \mathrm{r}$ simulations. ${ }^{4}$ Note that the $0-1-, 0-3-$, and $0-6-\mathrm{km}$ shear vector orientations remain constant across all simulations in this matrix. Though lifted condensation levels (LCLs) are another environmental parameter shown to discriminate between tornadic and nontornadic supercells (Brooks et al. 2003; Markowski and Richardson 2014), all of the basestate environments here are fairly moist throughout the depth of the troposphere and exhibit low LCLs, as is

\footnotetext{
${ }^{4}$ These changes result from the chosen effective shear-based storm motion estimate (Bunkers et al. 2014), which was used to be consistent with typical analysis and model fields. Actual storm motions between the simulations show minor differences, so true $\mathrm{SRH}$ is approximately the same.
} 


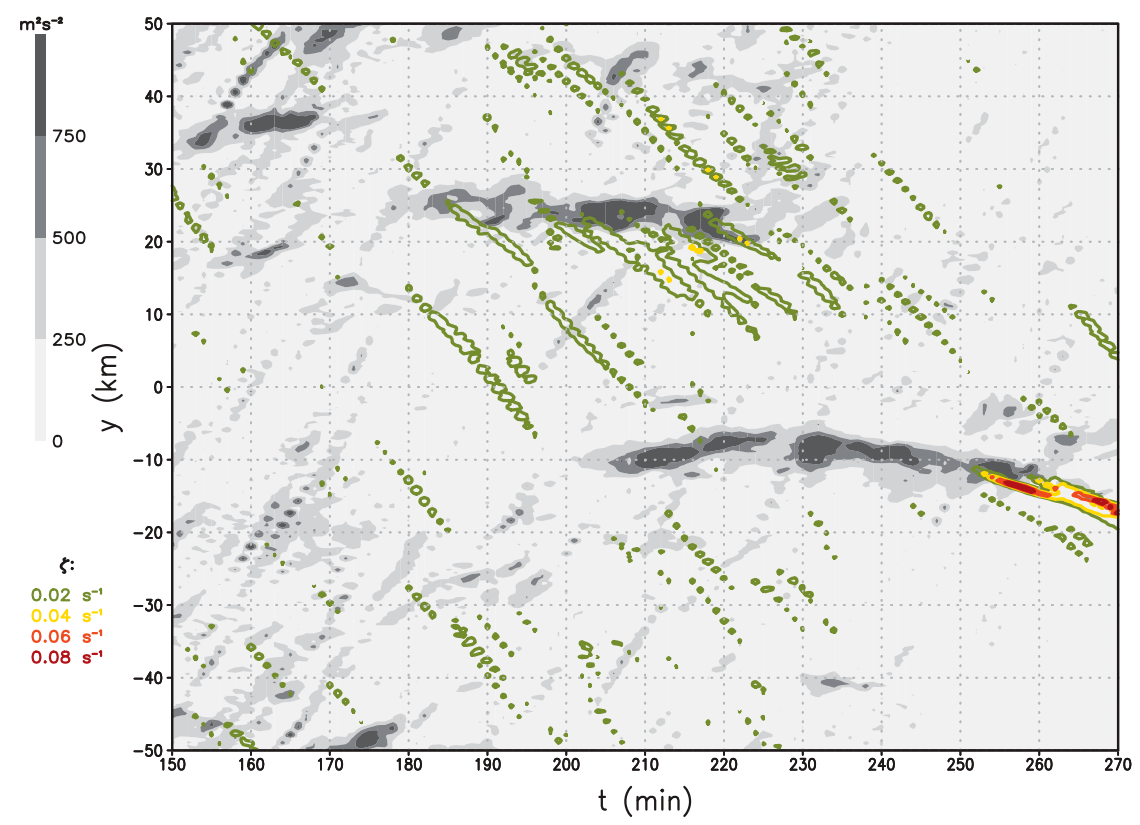

FIG. 5. Hovmöller plot of 2-5-km updraft helicity $\left(\mathrm{m}^{2} \mathrm{~s}^{-2}\right.$; shaded) and near-surface $\zeta\left(\mathrm{s}^{-1}\right.$; contours) tracks for the control simulation over the time period $t=150-270 \mathrm{~min}$.

typical in cool-season HSLC environments (e.g., Schneider et al. 2006; Sherburn et al. 2016).

Simulations were performed with the Bryan Cloud Model (CM1; Bryan and Fritsch 2002; Bryan and Rotunno 2009), release 18. Because prior work indicated the majority of HSLC severe events were associated with strong synoptic forcing and an associated boundary (e.g., Sherburn et al. 2016), simulations were initialized with a cold pool meant to mimic a cold front or outflow boundary. The cold pool was characterized by a minimum potential temperature perturbation of $-10 \mathrm{~K}$, decreasing as a cosine function eastward $260 \mathrm{~km}$ from the western edge of the domain and upward $6 \mathrm{~km}$ from the bottom edge of the domain. Although vertical motion due to synoptic-scale and mesoscale heterogeneity is not directly included in this idealized framework, this initiating cold pool is meant to represent linear forcing similar to an outflow boundary or cold front, along which HSLC severe convection tends to form and evolve. By virtue of the cold pool's structure, realistic destabilization through cooling aloft occurs east of the surface cold pool, leading to rather organic development and evolution of convection. Increased destabilization occurs with stronger low-level winds (i.e., increased 01s) due to increased advection and mixing. Other conventional initiation mechanisms, such as warm bubbles and forced convergence, were attempted but failed to produce sustained convection with the chosen base states. Further, sensitivity tests revealed that weaker initial cold pools would not support sustained convection. Thus, based on these simulations, it appears that HSLC environments are not very amenable to convection in the absence of forcing.

Horizontal grid spacing was $250 \mathrm{~m}$, with the $x$ domain stretched outside of the inner $100 \mathrm{~km}$ to lower the computational demand. The horizontal grid spacing here is fairly coarse given the scale of HSLC vortices (e.g., Davis and Parker 2014), and simulation results here are presented with the caveat that surface vortices are not entirely resolved. However, the focus here is on the processes leading to the development of these vortices, which should be reasonably represented given that comparable grid spacing has been used in prior similar studies (e.g., Dahl et al. 2014). Additionally, through preliminary tests, it was determined that there is little difference in storm structure or characteristics when the horizontal grid spacing is cut in half to $125 \mathrm{~m}$.

The vertical grid spacing was stretched from $10 \mathrm{~m}$ at the lowest model level $(5 \mathrm{~m})$ to $250 \mathrm{~m}$ from $9.875 \mathrm{~km}$ to the top of the domain. The domain moved with a constant speed that varied slightly depending upon the basestate hodograph to ensure that convection remained near the center of the domain. Boundaries were open in the $x$ direction and periodic in the $y$ direction. Coriolis forcing was included on the perturbation winds only, which is equivalent to assuming geostrophic balance in the base-state wind field (Roberts et al. 2016; Coffer and Parker 2017). The simulations were initialized with modest $( \pm 0.25 \mathrm{~K}$ or smaller), pseudorandom potential temperature perturbations throughout the domain to encourage development of three-dimensional convective structures. Sensitivity tests varying the distribution 

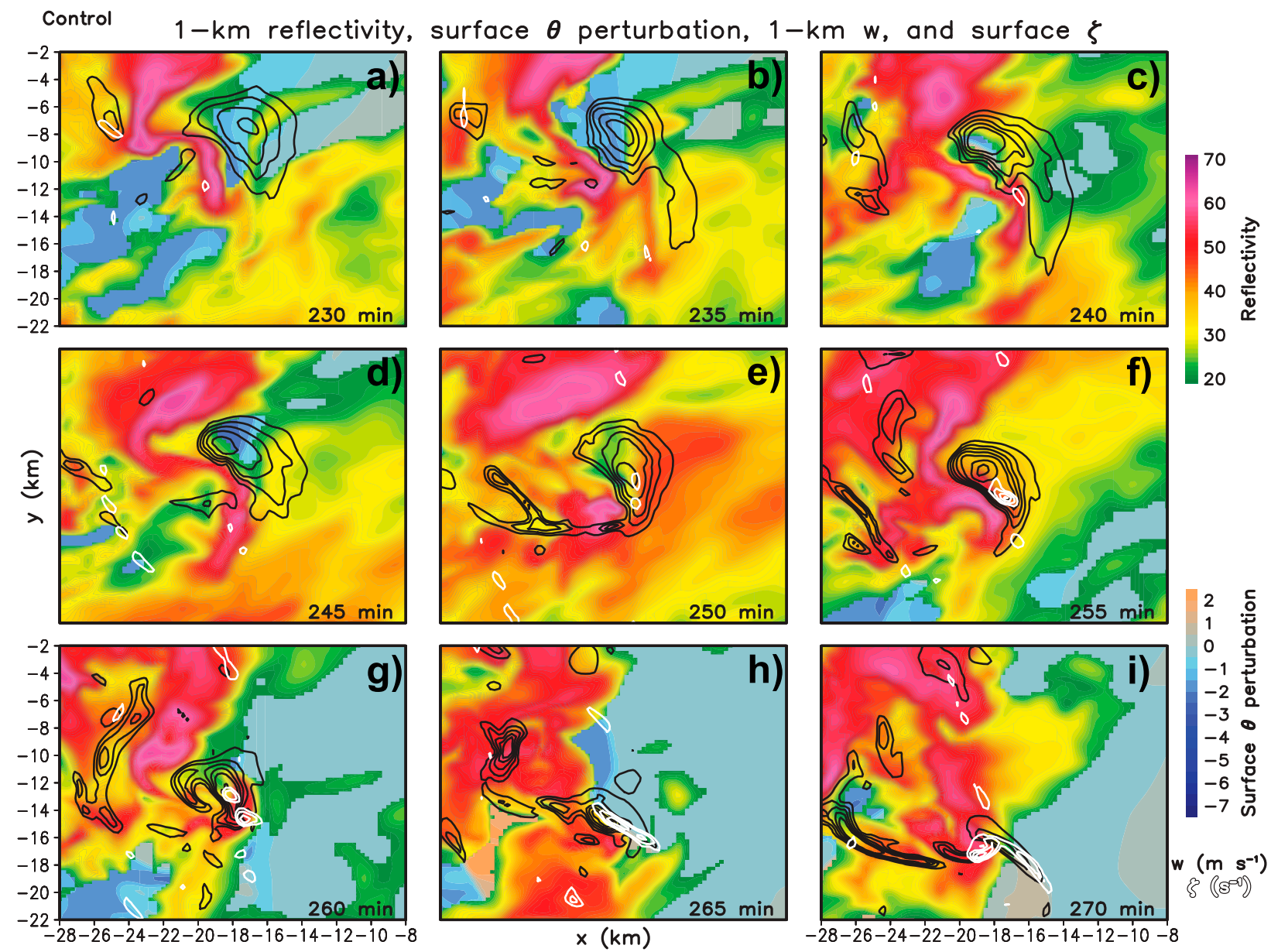
30

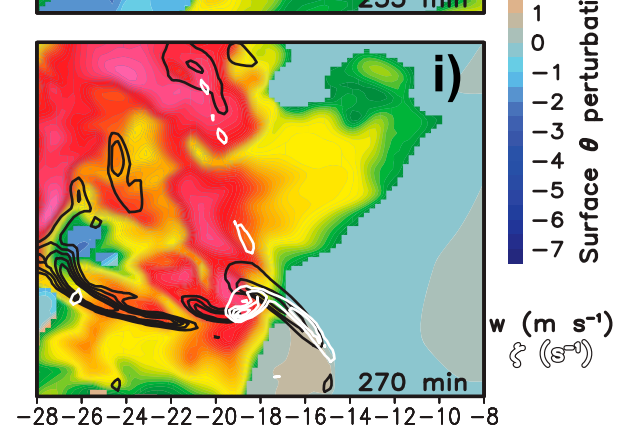

FIG. 6. As in Fig. 4, but for zoomed-in plot for the control simulation's strongest updraft from (a) $t=230 \mathrm{~min}$ through (i) $t=270 \mathrm{~min}$ over 5-min intervals. Note that this takes place in the area of $x=-28,-8$ and $y=-22,-2$ for comparison with Figs. 4 and 5.

of these perturbations showed little change in resulting convection's mode or characteristics. The NSSL doublemoment microphysics scheme (Mansell et al. 2010) was used, with both graupel and hail densities predicted.

Many of the choices in the model setup were made for simplicity, in order to focus on the processes of primary interest, or to reduce computational demand. For example, surface fluxes of heat and moisture-which are thought to influence HSLC environments only modestly (e.g., King et al. 2017) — and radiation are excluded for simplicity. Additionally, the bottom boundary in this matrix of simulations is free-slip, consistent with the majority of prior QLCS mesovortexgenesis and supercell tornadogenesis studies. Nonetheless, surface drag could potentially influence the development of surface vortices or otherwise modify the near-surface wind field (e.g., Schenkman et al. 2012, 2014; Markowski 2016; Roberts et al. 2016). Thus, one additional simulation was conducted with a semislip bottom boundary condition, which produced some differences in surface vortex characteristics compared with the results explored here. However, there remains considerable uncertainty regarding how surface drag should realistically be included within idealized convection simulations, particularly in the absence of a turbulent boundary layer (e.g., Markowski and Bryan 2016; Markowski 2018). Therefore, we view these free-slip simulations as a first attempt at modeling critical processes leading to tornadogenesis in HSLC environments and hope that a clearer idea of the proper way to formulate a semislip bottom boundary condition in idealized runs will help us add sophistication in future work. Future work should also take advantage of finer grid spacing to assess how the important processes examined here are affected at higher resolution and to begin to study the details of the vortex dynamics. Additionally, as noted above, a more realistic boundary layer including surface drag could be utilized to assess the role of neglected nearground processes in the vortexgenesis process, though this should only be included at scales capable of resolving turbulent eddies to prevent unrealistic low-level shear 

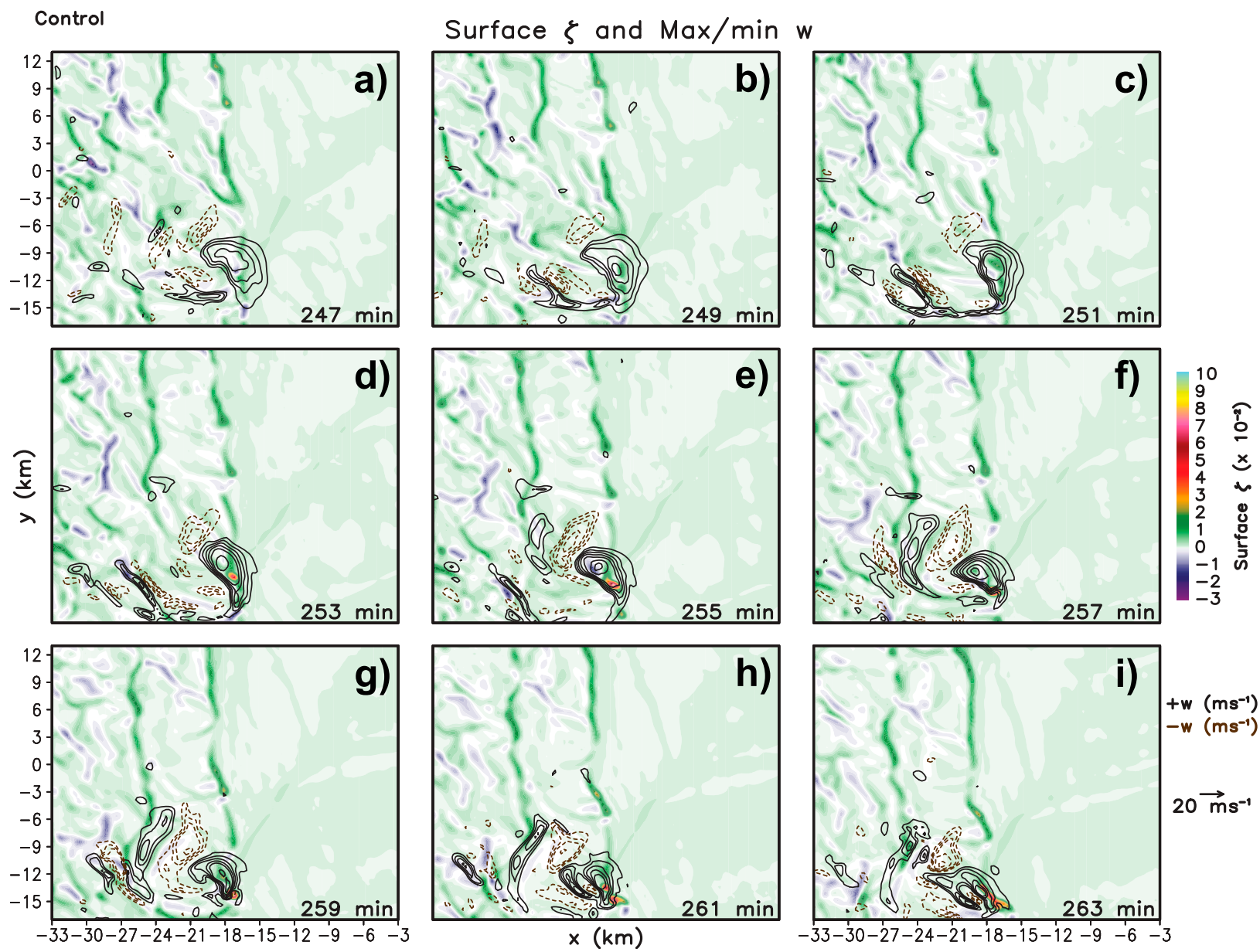

FIG. 7. Surface $\zeta\left(\mathrm{s}^{-1}\right.$; rainbow shading) and maximum (black contours) and minimum (brown contours) $w$ in the lowest $1 \mathrm{~km}\left(\mathrm{~m} \mathrm{~s}{ }^{-1}\right)$ for the control simulation's strongest updraft from (a) $t=247 \mathrm{~min}$ through (i) $t=263 \mathrm{~min}$ over 2-min intervals.

profiles (Markowski and Bryan 2016). Despite these caveats, the given model setup appears to reasonably depict the basic evolution of HSLC convection that ultimately fosters the development of embedded vortices based on comparisons to observed cases.

\section{b. Object tracking algorithm}

To analyze the characteristics of low-level updrafts and vortices (i.e., those in the lowest $0.5-2 \mathrm{~km}$ AGL) within each simulation, an algorithm to objectively detect and track these features was developed. 3D fields were compressed to $2 \mathrm{D}$ by taking the maximum value of either $w$ (for updrafts) or the Okubo-Weiss parameter (OW, for vortices; Okubo 1970; Weiss 1991) ${ }^{5}$ over the

\footnotetext{
${ }^{5}$ The Okubo-Weiss parameter $\left\{\mathrm{OW}=[(d v / d x)-(d u / d y)]^{2}-\right.$ $\left.[(d u / d x)-(d v / d y)]^{2}-[(d v / d x)-(d u / d y)]^{2}\right\}$ is sometimes preferred over $\zeta$ because it effectively removes deformation from $\zeta$, thus providing a focus on the location where rotation (as opposed to shearing) is dominant.
}

lowest $1.5 \mathrm{~km}$. Then, an object detection tool was used to identify 2D closed contours within which $w \geq 5 \mathrm{~m} \mathrm{~s}^{-1}$ or $\mathrm{OW} \geq 0.001 \mathrm{~s}^{-2}$ was satisfied over an area of at least $1.5 \mathrm{~km}^{2}$. In addition to identifying updraft and vortex features meeting these thresholds, the algorithm was able to objectively determine the maximum and mean values of the chosen fields along with the area and centroid of the features. The objects were subject to a minimum depth criterion: threshold values of $w$ (or OW) had to be met over 12 (8) grid points in the lowest $1.5 \mathrm{~km}$ to be considered. These criteria were incorporated to prevent the presence of small, inconsequential features in the population of updrafts and vortices. Several other criteria thresholds were also tested, and while updraft and vortex counts did vary, relative distribution characteristics were similar across all thresholds.

Once features were detected, the algorithm searched the same grids $1 \mathrm{~min}$ later to determine the new location of these features. The algorithm searched within a 10 -grid 

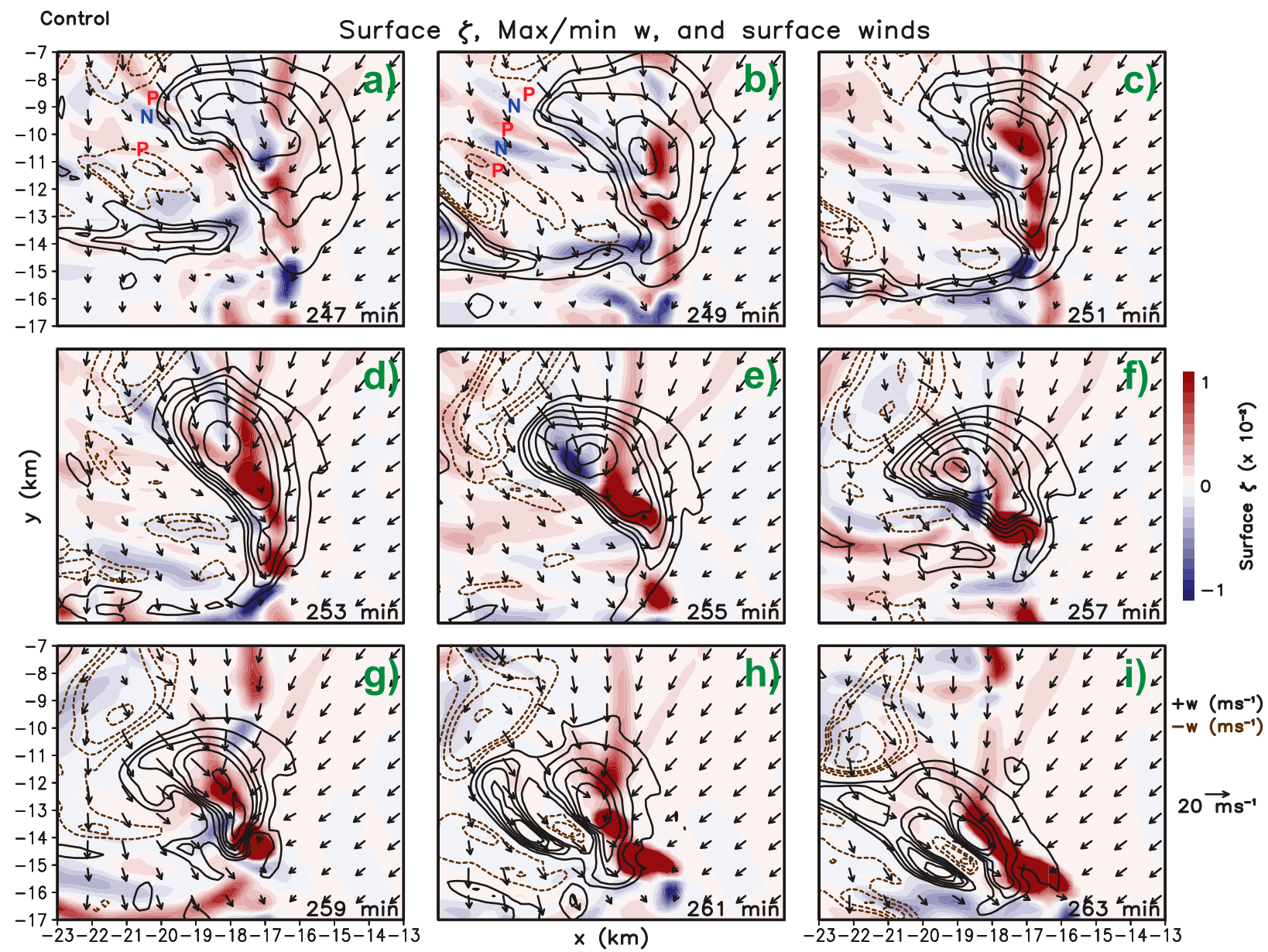

FIG. 8. As in Fig. 7, but zoomed-in and including lowest model level wind vectors $\left(\mathrm{m} \mathrm{s}^{-1}\right)$. Note change in color bar meant to highlight lower values of vertical vorticity. Red "P"s and blue "N"s in first two panels show alternating positive and negative bands of vertical vorticity appearing to emanate from the downdraft.

point (4-grid points for vortices) box surrounding the feature's centroid during the previous output time, starting with the grid point nearest the prior centroid. Because the domain was moving at a speed generally close to that of the convection, the centroid tended to move little between time steps; thus, features of interest were tracked readily. Features were subsequently subject to a longevity requirement of $5 \mathrm{~min}$, as transient updrafts and vortices were not the intended subject of analysis.

By detecting and tracking updrafts and vortices, it was possible to analyze the distributions of these featuresincluding their typical sizes, intensities, and durationsand how their characteristics varied across environments. The distributions of values for a population of objectively identified objects over time were much more representative of the environmental sensitivities than a single maximum value, the latter of which was at times inconclusive or even misleading.

\section{c. Analysis of accelerations}

To isolate cause from effect in these sensitivity experiments, we seek to quantify the specific impacts of $01 \mathrm{~s}$ and 031r upon the low-level updrafts of the simulated storms. Regardless of convective mode, a strong lowlevel vortex cannot develop and be maintained without low-level stretching of $\zeta$, which is driven by low-level vertical accelerations. Thus, careful analysis of low-level accelerations was a primary focus of this study. When the Boussinesq approximation is valid and frictional and viscous forces are ignored, vertical accelerations are governed by the following expression:

$$
\frac{D w}{D t}=-\frac{1}{\rho_{0}} \frac{\partial p^{\prime}}{\partial z}+B
$$

where the first term on the right-hand side is the vertical perturbation pressure gradient acceleration, and the second term is the buoyancy. Vertical accelerations can 

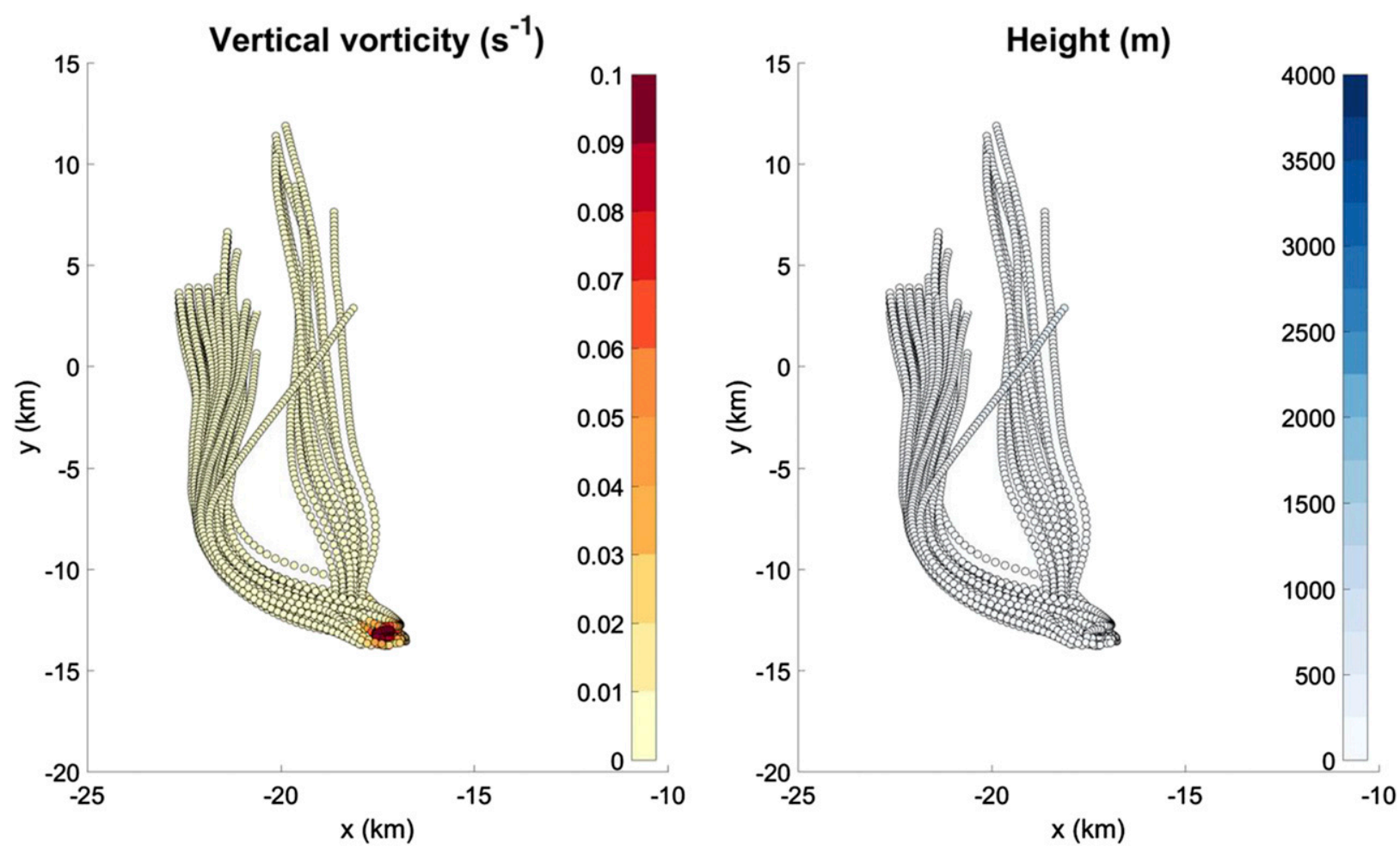

FIG. 9. Trajectories, showing (left) $\zeta\left(\mathrm{s}^{-1}\right)$ and (right) height $(\mathrm{m})$ of parcels contributing to a strong, near-surface vortex in the control simulation. Note that each parcel's trajectory trace ends approximately when that parcel enters the vortex to prevent confusion about whether the parcel is entering or exiting the vortex. Time over which trajectories are plotted is $240-256.5 \mathrm{~min}$.

subsequently be broken down into buoyant and dynamic components (Wilhelmson and Ogura 1972; Rotunno and Klemp 1982), which involves decomposing the total pressure perturbation $\left(p^{\prime}\right)$ into its buoyant $\left(p_{b}^{\prime}\right)$ and dynamic $\left(p_{d}^{\prime}\right)$ components.

The buoyant term, given by

$$
\alpha_{0} \nabla^{2} p_{b}^{\prime}=\frac{\partial B}{\partial z}
$$

tends to be small in low-CAPE environments, as a lifted parcel has a density relatively close to that of the ambient environment. Therefore, vertical accelerations resulting from the dynamic perturbation pressure gradient likely dominate in HSLC convection (e.g., McCaul and Weisman 1996; Markowski and Richardson 2014). The dynamic component of the perturbation pressure is given by

$$
\begin{aligned}
\nabla^{2} p_{d}^{\prime}= & -\rho_{0}\left[\left(\frac{d u}{d x}\right)^{2}+\left(\frac{d v}{d y}\right)^{2}+\left(\frac{d w}{d z}\right)^{2}\right]-w^{2} \frac{\partial^{2}}{\partial z^{2}}\left(\ln \rho_{0}\right) \\
& -2 \rho_{0}\left(\frac{d v}{d x} \frac{d u}{d y}+\frac{d u}{d z} \frac{d w}{d x}+\frac{d v}{d z} \frac{d w}{d y}\right)
\end{aligned}
$$

where $u, v$, and $w$ are $3 \mathrm{D}$ wind components and $\rho_{0}$ is density. The linear component of $p_{d}^{\prime}$ incorporates effects of an updraft interacting with the vertical wind shear vector (Rotunno and Klemp 1982; Klemp 1987). It is referred to here as $p_{\mathrm{dl}}^{\prime}$ and is given by

$$
\nabla^{2} p_{\mathrm{dl}}^{\prime}=-2 \rho_{0}\left(\frac{d u_{0}}{d z} \frac{d w}{d x}+\frac{d v_{0}}{d z} \frac{d w}{d y}\right)
$$

where $u_{0}$ and $v_{0}$ are the base-state horizonal wind components. The nonlinear terms in Eq. (2.3) are then combined as $p_{\mathrm{dnl}}^{\prime}$, which is equivalent to $p_{d}^{\prime}-p_{\mathrm{dl}}^{\prime}$ and represents deformation and rotation due to perturbation winds (Davies-Jones 2002). The pressure perturbation equations given above were solved following a similar methodology to Parker and Johnson (2004a,b), Parker (2007, 2010, 2017), Davenport and Parker (2015), and Coffer and Parker (2015, 2017). In terms of the three components, the equation for vertical accelerations is then

$$
\frac{D w}{D t}=\underbrace{B-\frac{1}{\rho_{0}} \frac{\partial p_{b}^{\prime}}{\partial z}}_{\mathrm{ACCB}}-\underbrace{\frac{1}{\rho_{0}} \frac{\partial p_{\mathrm{dl}}^{\prime}}{\partial z}}_{\mathrm{ACCDL}}-\underbrace{\frac{1}{\rho_{0}} \frac{\partial p_{\mathrm{dnl}}^{\prime}}{\partial z}}_{\mathrm{ACCDNL}},
$$

where $B$ includes the effects of hydrometeor loading.

Within supercells, nonlinear dynamic accelerations (ACCDNL) can substantially augment vertical 

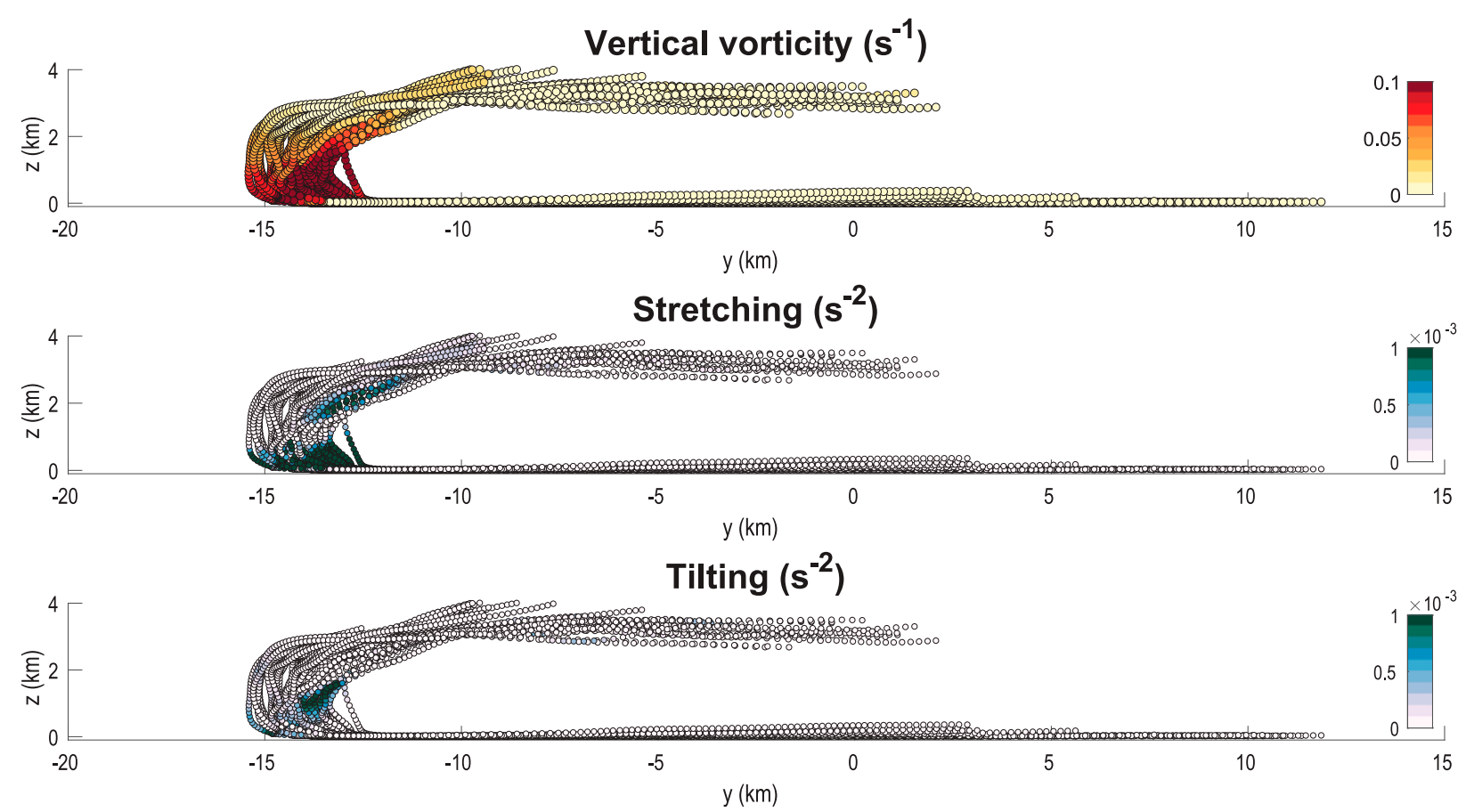

FIG. 10. As in Fig. 9, but for the $y-z$ plane and showing stretching and tilting $\left(\mathrm{s}^{-2}\right)$ rather than height. Also note that this figure represents a longer time period (240-270 min) than Fig. 9 (including the time after parcels exit the vortex).

velocities in the updraft and have been shown to be the primary contributor to vertical accelerations in numerical studies of supercells (e.g., Weisman and Klemp 1984; McCaul and Weisman 1996; Weisman and Rotunno 2000; Coffer and Parker 2017). Because lowlevel shear vector magnitude plays a significant role in the strength of low-level mesocyclones in supercells (Brooks et al. 2003; Adlerman and Droegemeier 2005; Markowski and Richardson 2014; Coffer and Parker 2015, 2017), low-level ACCDNL could be significant in supercellular HSLC convection.

Rather than strictly focusing on the origins and lifetimes of strong surface vortices in these simulations, much of the analysis will instead focus on the origins of strong, low-level updrafts that facilitate and maintain these vortices. The reasoning for this choice is twofold: 1) As noted throughout the manuscript thus far, the most important consideration in surface vortex strength is the ability for vorticity near the surface to be tilted and stretched, a process made possible through a strong low-level updraft; and 2) convective updrafts are well resolved on the chosen model domain, while vortices are only marginally resolved on the 250-m horizontal grid. This analysis will lead to an understanding of the processes that precede the intensification of surface vortices and will allow for the identification of any storm-scale precursors that could be observed in real cases.

\section{d. Parcel trajectory analysis}

For each primary matrix simulation, a restart run was performed in which tracer parcels were seeded within the model. Parcels were initiated at each grid point within a $50 \mathrm{~km}$ (in $x$ ) by $100 \mathrm{~km}$ (in $y$ ) by $1.4 \mathrm{~km}$ (in $z$ ) box ahead, and in the vicinity, of the location where the strongest or longest-lived updraft developed, and then integrated forward in time. Parcel trajectories were calculated at every large model time step, with outputcalculated via trilinear interpolation of $3 \mathrm{D}$ model fields-written every $10 \mathrm{~s}$ in the simulation. Candidate parcels entering low-level updrafts and surface vortices were then identified to determine the origins and key accelerations contributing to the strongest features. These trajectories offered insight into the Lagrangian characteristics of the parcels that contributed to strong updrafts and vortices-particularly how the accelerations affecting their motion changed as they approached and entered the updrafts-while also allowing for comparisons of these characteristics among the environmental sensitivity simulations.

\section{Results}

\section{a. Overview of control simulation}

Convection in the control simulation was fairly slow to develop, with appreciable updrafts (i.e., $w \geq 10 \mathrm{~m} \mathrm{~s}^{-1}$ ) 
first appearing nearly $2 \mathrm{~h}^{6}$ after the model was started. Convective updrafts tended to originate within $10 \mathrm{~km}$ of the leading edge of the initiating cold pool (e.g., Fig. 4a), which retreated westward relative to the domain's motion prior to the development of convection. Convective evolution proceeded rapidly, with upscale growth into a QLCS occurring over the next 30-60 min (Figs. 4b,c). Convection then progressively moved east of the initiating boundary due to the ambient environmental flow and the establishment of a system-generated cold pool (Figs. 4d-i).

Beyond $3 \mathrm{~h}$, embedded rotating updrafts became apparent, as shown in Hovmöller diagrams of 2-5-km updraft helicity and surface (lowest model level) $\zeta$ (Fig. 5; also visible in Figs. 4c-i where updraft and $\zeta$ contours overlap). These features correspond to embedded supercells within the predominant QLCS mode. Rotating updrafts are supported by the favorable lower and middle tropospheric wind profile, with the majority of parcels contributing to these updrafts arriving from the lowest $500 \mathrm{~m}$ AGL and acquiring rotation via tilting of environmental vorticity, followed by stretching (not shown). In addition to rotating updrafts, numerous weak surface vortices became established along the leading edge of the system-generated cold pool (green contours in Fig. 5) and move southward relative to the storm motion, consistent with flow within the cold pool. The majority of these vortices originated within or near embedded supercell downdrafts and strengthened as they move into regions of convergence or overlying updraft near the leading edge of the system-scale outflow boundary. Subsequently, the vortices weakened as they moved southward out of the region of enhanced updraft and convergence near the embedded supercell. New updrafts occasionally developed near and just ahead of this QLCS, including the strongest updraft of the simulation that became dominant just prior to $4 \mathrm{~h}$ into the simulation (Fig. 6; note strong, relatively steady updraft in Figs. 6a-g and development of surface vortex in Figs. 6d-i). This updraft, which was subsequently ingested into the QLCS, exhibited persistent rotation and a hook echo and eventually supported a series of strong surface vortices (Fig. 5) before weakening.

Over the last $30 \mathrm{~min}$ of the control simulation, positive $\zeta$ is essentially ubiquitous along the cold pool's leading edge north of the dominant rotating updraft (Fig. 7). This also occurred in a sensitivity run excluding Coriolis, though to a lesser degree. Additional, subtle maxima and minima of $\zeta$ appear to be emanating from the convective downdrafts (Fig. 8), though positive $\zeta$ generally dominated.

\footnotetext{
${ }^{6}$ This took longer than expected, but given extremely limited instability, the environmental evolution that provided organic development of convection was deemed important and worth the upfront computational demand.
}
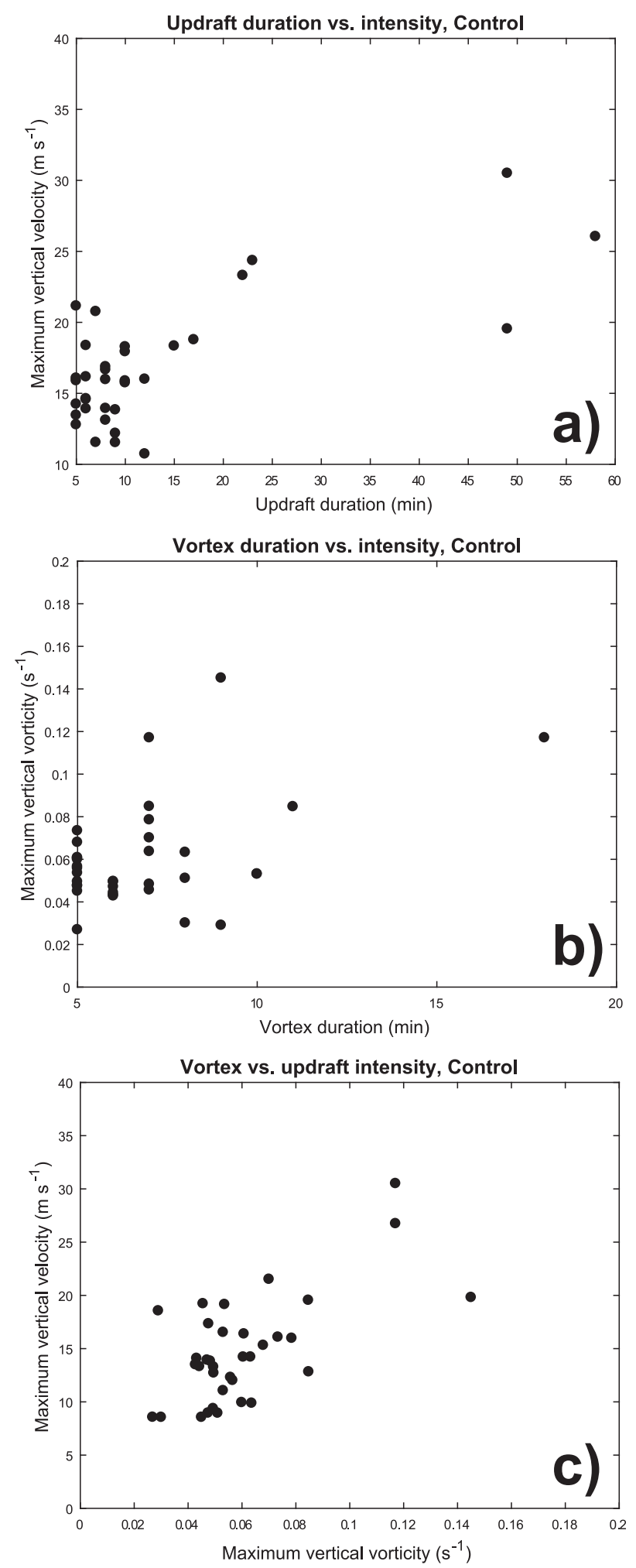

FIG. 11. Scatterplot of (a) updraft duration (min) vs maximum $w\left(\mathrm{~m} \mathrm{~s}^{-1}\right)$, (b) vortex duration (min) vs maximum $\zeta\left(\mathrm{s}^{-1}\right)$, and (c) maximum $\zeta\left(\mathrm{s}^{-1}\right)$ vs maximum $w\left(\mathrm{~m} \mathrm{~s}^{-1}\right)$ for each updraft or vortex object tracked for at least $5 \mathrm{~min}$ in the control simulation. 

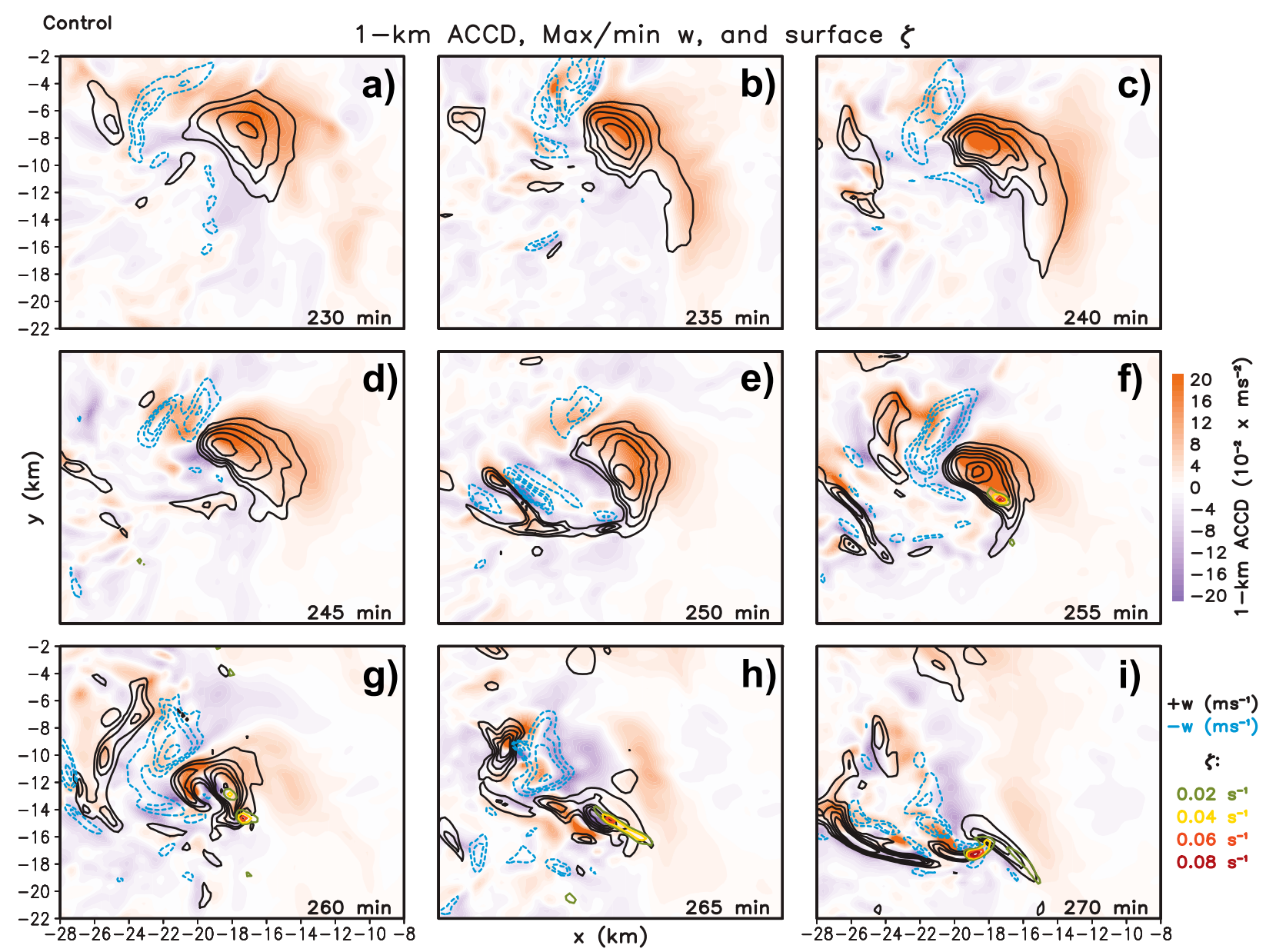

FIG. 12. As in Fig. 6, but showing 1-km ACCD $\left(\times 10^{-2} \mathrm{~m} \mathrm{~s}^{-2}\right.$; shading), surface-to-1-km maximum (black contours) and minimum (blue contours) $w\left(\mathrm{~m} \mathrm{~s}^{-1}\right)$, and lowest level $\zeta\left(\mathrm{s}^{-1}\right.$; green to red contours).

In terms of their basic positioning, the two zones of $\zeta$ within the embedded supercell somewhat resemble the "rivers" feeding a near-surface vortex in a high-CAPE supercell simulated by Dahl et al. (2014). Trajectory analysis confirms that there are indeed two primary source regions for the air parcels that are converged into a strong vortex (Fig. 9; note that each parcel's trace ends when that parcel enters the vortex).

Parcels initially contributing to the strong vortex in the control run had limited $\zeta$ (i.e., $\leq 0.01 \mathrm{~s}^{-1}$ ) as they approached the updraft from the two aforementioned source regions (Fig. 9, left and Fig. 10, top). In the $10-15 \mathrm{~min}$ prior to contributing to the vortex, these parcels approached the updraft along or very near the bottom model level (Figs. 9 and 10). As in prior supercell tornadogenesis studies, stretching ${ }^{7}$ was the

\footnotetext{
${ }^{7}$ Stretching is defined as $\zeta(d w / d z)$, while tilting is defined as $\xi(d w / d x)+\eta(d w / d y)$.
}

primary contributor to the rapid development of $\zeta$ as the parcels ascended within updraft (Fig. 10, cf. middle and bottom). The $\zeta$ typically exceeded $0.1 \mathrm{~s}^{-1}$ by an altitude of $180 \mathrm{~m}$, and the vast majority of stretching (and thus enhancement of $\zeta$ ) occurred in the lowest 0.5 to $1 \mathrm{~km}$ above ground (Fig. 10, middle), providing evidence that strong low-level rotating updrafts were critical for the intensification of surface HSLC vortices here.

Given the importance of stretching, it is worthwhile to isolate and study the strongest low-level updrafts that occur in the control simulation. The updraft tracking algorithm identified over 30 low-level updrafts that persisted for five or more minutes in the control simulation (Fig. 11a). Five of these had lifetimes of over $20 \mathrm{~min}$, including the strongest that is explored in more detail throughout this section. The mean maximum intensity for these updrafts in the lowest $1.5 \mathrm{~km}$ was approximately $18 \mathrm{~m} \mathrm{~s}^{-1}$, while the mean duration was about $12 \mathrm{~min}$. Additionally, 35 low-level (lowest $1.5 \mathrm{~km} \mathrm{AGL}$ ) 

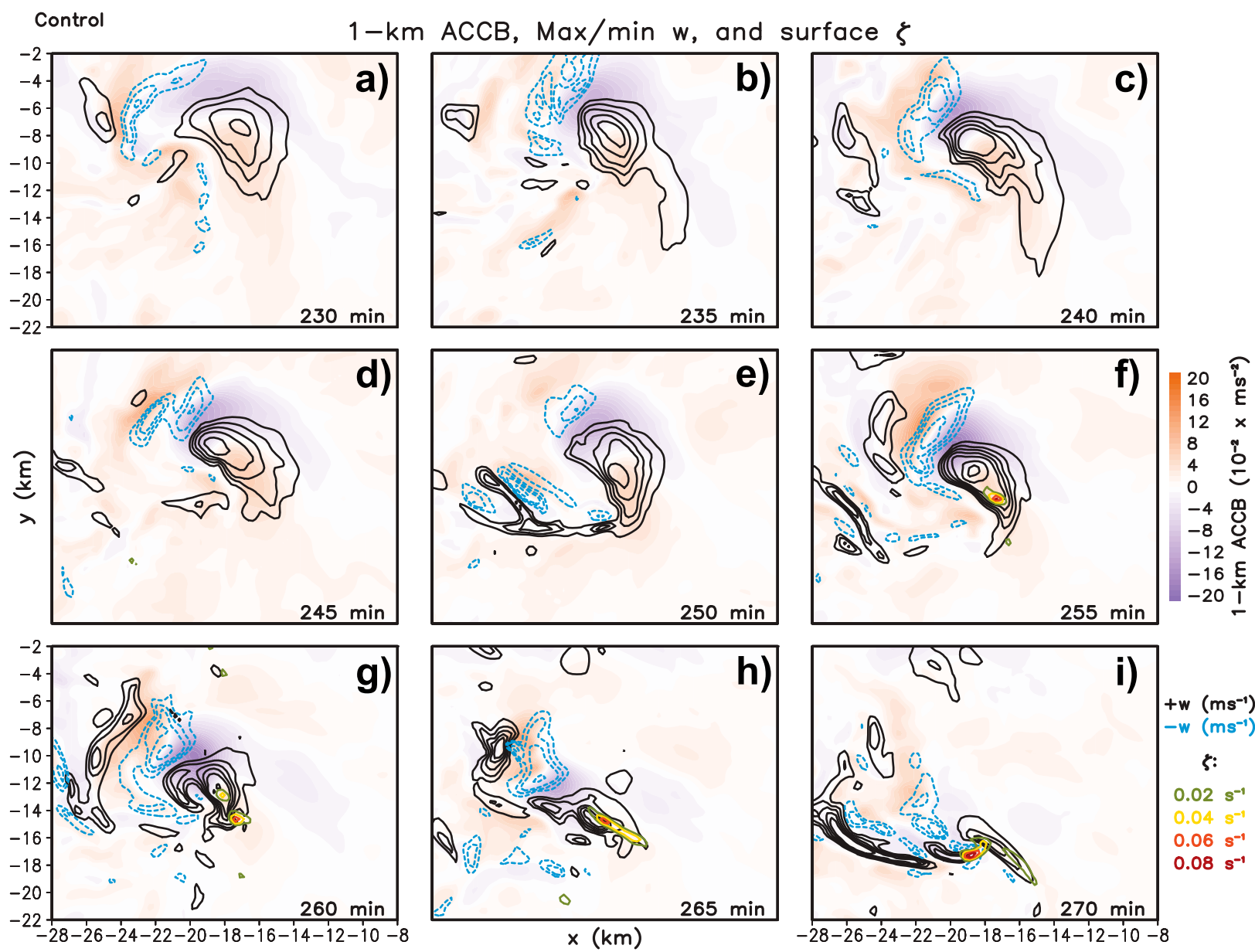

FIG. 13. As in Fig. 12, but for 1-km ACCB $\left(\times 10^{-2} \mathrm{~m} \mathrm{~s}^{-2}\right.$; shading $)$.

vortices were identified in the control simulation (Fig. 11b). These tended to be a bit shorter lived than updrafts, with a typical duration of approximately $7 \mathrm{~min}$ for the control run. The short vortex durations are unsurprising, given operational evidence of rapid strengthening and demise of HSLC vortices (e.g., Cope 2004). However, one longer-lived vortex with a lifetime of $18 \mathrm{~min}$ was also identified. The strongest vortices were not necessarily the longest lived; instead, vortex strength again appeared to be more dependent upon the strength of the overlying low-level updraft (Fig. 11c). Additionally, the strongest vortices tended to be those that originated at the surface, then grew upward via stretching. This provides further support for the contention that the strength of lowlevel updrafts appears to be a considerable factor in the potential intensification of surface vortices (e.g., Markowski and Richardson 2014). Note that many of the updrafts tracked here are nonsupercellular given their longevity and intensity. The strongest and longest-lived updrafts, however, exhibit persistent rotation and are clearly supercellular, suggesting that rotation may be required to support a long-lived updraft. The forcing mechanisms for the strongest lowlevel updraft will be explored next.

\section{b. Vertical accelerations and the processes leading to surface vortexgenesis}

For the purposes of this discussion, we will focus on the strongest updraft in the control simulation, which was associated with an embedded supercell and supported the longest-lived vortex. Focusing on one particular updraft allows for a clear depiction and discussion of the processes that lead to surface vortexgenesis. However, this updraft was not the only strong updraft in the simulation. Within the control run, several low-level updrafts exceeded $20 \mathrm{~m} \mathrm{~s}^{-1}$. Given that these values existed very close to the height of the LFC, it is likely that any buoyant accelerations were augmented by other processes. Indeed, within mature updrafts, positive low-level accelerations attributable to the dynamic perturbation pressure gradient acceleration 

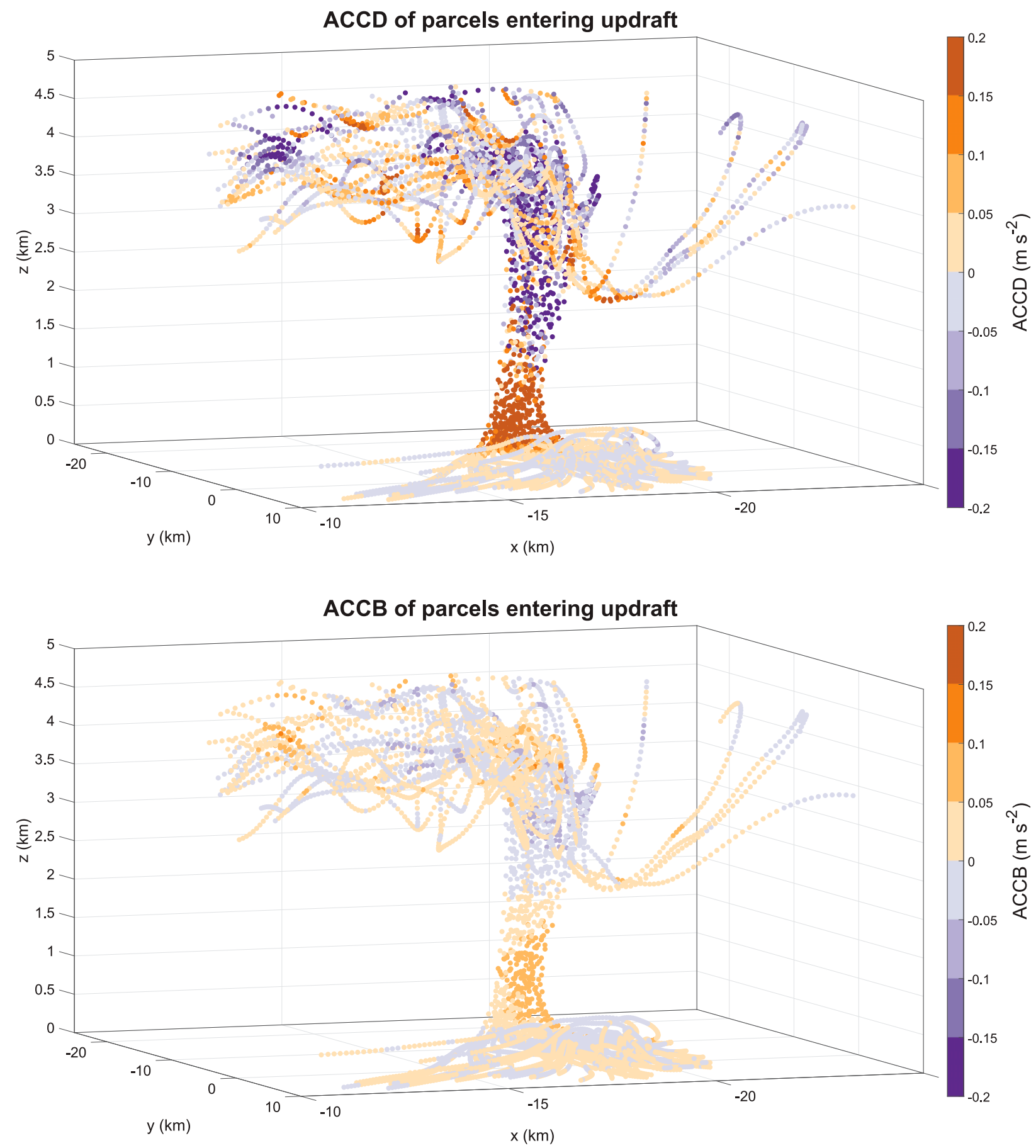

FIG. 14. Trajectories showing (top) ACCD $\left(\mathrm{m} \mathrm{s}^{-2}\right)$ and (bottom) ACCB $\left(\mathrm{m} \mathrm{s}^{-2}\right)$ of parcels entering the strongest updraft in the control simulation, looking south-southwest. Time period plotted is $240-270 \mathrm{~min}$.

components (ACCD; Fig. 12) appear to be more critical for low-level accelerations than the total buoyant acceleration (ACCB), which is generally more modest (Fig. 13). Additionally, ACCD maxima relate well with the strongest embedded updraft (Fig. 12). This was further supported by examining the characteristics of parcels entering the updraft, the accelerations for which were dominated by ACCD when compared to ACCB (Fig. 14). Parcels approaching the updraft exhibited predominantly horizontal motion prior to reaching the region of strong upward-pointing ACCD, confirming that this acceleration 

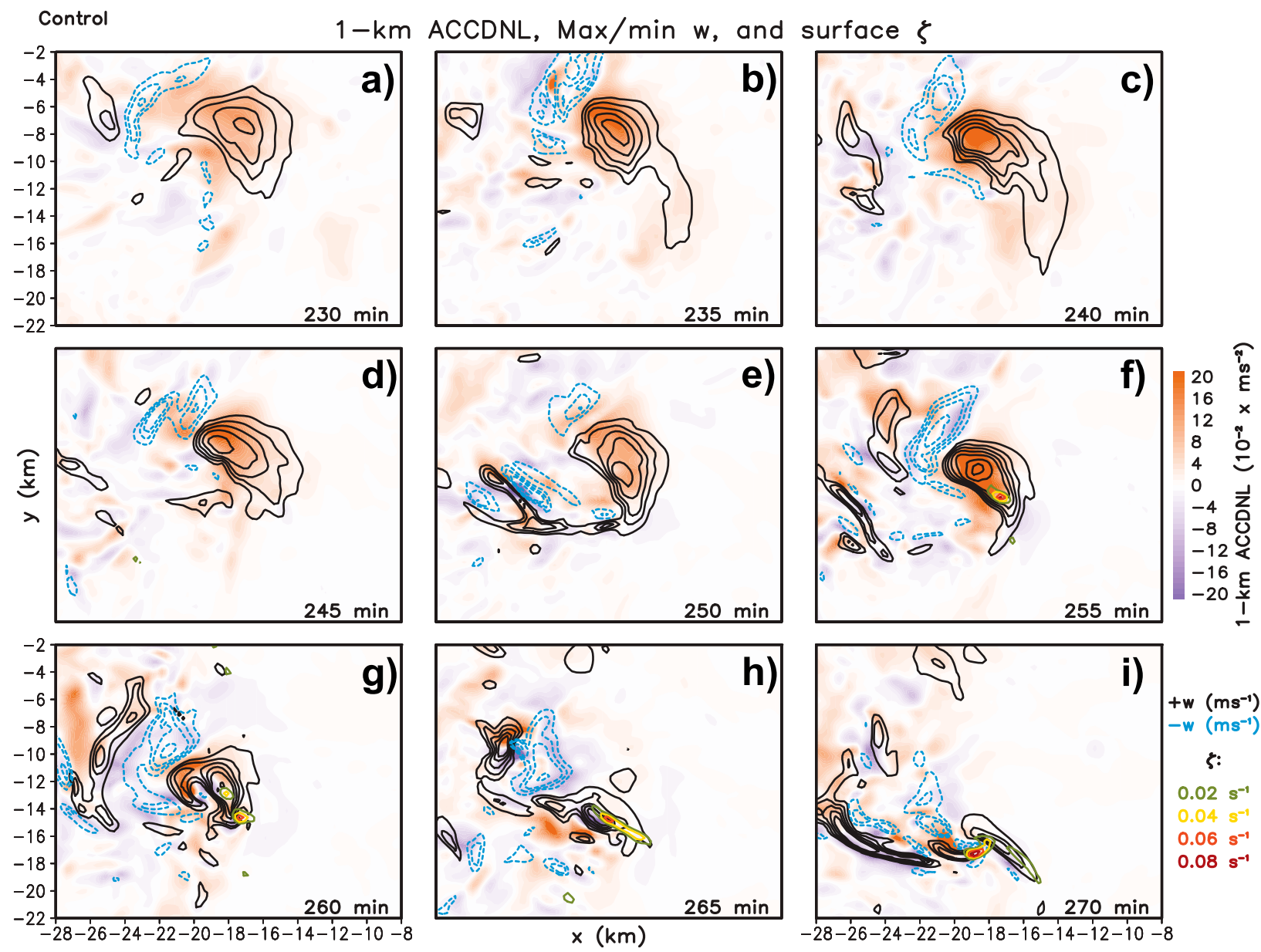

FIG. 15. As in Fig. 12, but for 1-km ACCDNL $\left(\times 10^{-2} \mathrm{~m} \mathrm{~s}^{-2}\right.$; shading).

was necessary for the appreciable low-level updrafts (Fig. 14).

By breaking ACCD down into its linear (ACCDL) and nonlinear (ACCDNL) components, it becomes clear that the ACCDNL terms are most important for strength and maintenance of the core of this rotating updraft. ACCDNL maxima through time are associated well with the maxima in $w$ (Fig. 15), while ACCDL maxima and minima are weaker and generally straddle the maximum updraft (Fig. 16). These findings are in line with theory, given that high and low pressure perturbations attributable to $p_{\mathrm{dl}}^{\prime}$ develop on the upshear and downshear sides of the updraft, and those associated with $p_{\mathrm{dnl}}^{\prime}$ tend to be located within areas of rotation, which are maximized near the updraft. This finding is also consistent with prior investigations of supercell simulations (e.g., Weisman and Rotunno 2000; Markowski and Richardson 2014; Coffer and Parker 2017), including those in environments with limited CAPE (e.g., McCaul and Weisman 1996). Further, the general scales of these vortices are comparable to those in simulated high-CAPE supercells (e.g., Coffer and Parker 2017) and QLCSs (e.g., Atkins and St. Laurent 2009a) and are consistent with observed radar characteristics of HSLC vortices (Davis and Parker 2014). The spatial scales of the convection, in terms of updraft depth and width, appear to be smaller than in higher-CAPE environments, again consistent with observations. This sometimes leads to gray area in the distinction between low-level and midlevel features, as midlevel features would presumably be found closer to the ground in this relatively shallow convection.

Despite many similarities to tornadogenesis in discrete, high-CAPE supercells, the interior of this control QLCS seems to possess far more transient and rapidly evolving features on the system scale than the quasi-steady state, long-lived, discrete supercells that have historically been simulated and studied in tornadogenesis research. These transient features include numerous near-surface vortices that generally develop through similar means those that become longer-lived vortices but do not benefit from 

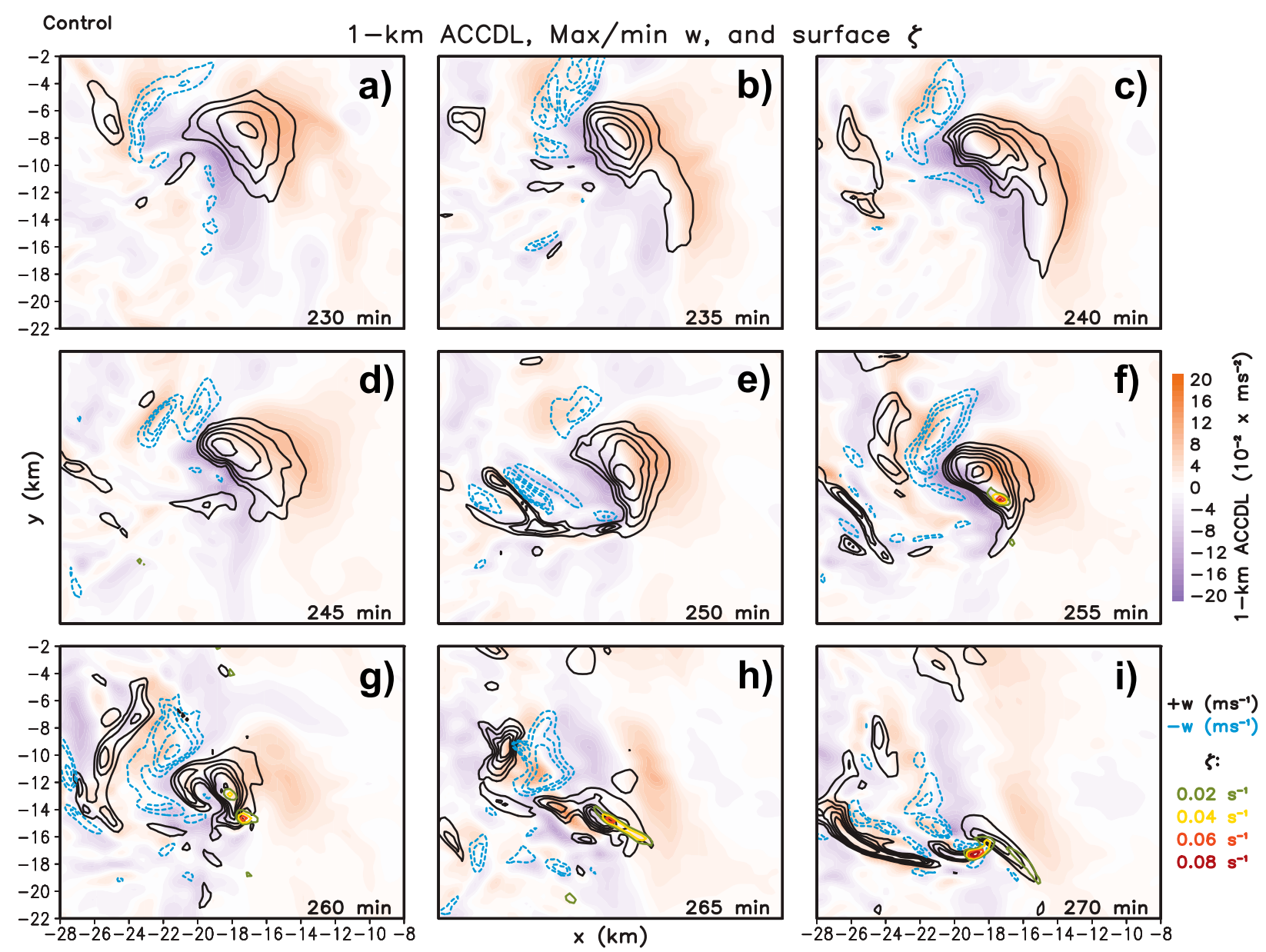

FIG. 16. As in Fig. 15, but for 1-km ACCDL $\left(\times 10^{-2} \mathrm{~m} \mathrm{~s}^{-2}\right.$; shading $)$.

favorable collocation with an overlying updraft and near-surface convergence zone. The comparatively transient nature of our embedded supercell may be associated with its location within - and interaction witha parent QLCS, which offers a larger number of source regions than a typical discrete supercell due to its multiple downdrafts. However, as simulations of high-CAPE tornadic supercells continue to benefit from increased spatial and temporal resolutions (e.g., Orf et al. 2017), it is progressively clearer that even purportedly steadystate storms exhibit many transient, rapidly evolving features similar to those in the simulations presented here.

In summary, we can offer the following list of processes that lead to near-surface vortexgenesis within an embedded supercell in the control run (Fig. 17):

1) Low- to midlevel $(0.5-2 \mathrm{~km}) \zeta$ begins to generally increase as the midlevel updraft strengthens (this is playing the role that a low-level mesocyclone would in a high-CAPE supercell);
2) in response to item 1, low-level, upward-pointing ACCDNL increases;

3) in response to item 2, low-level $w$ is enhanced, bringing strong updrafts closer to the ground; and

4) resulting from item 3 , the potential for tilting horizontal vorticity into the vertical and stretching $\zeta$ increases.

This chain of processes appears to begin several minutes prior to the development of the surface vortex. In fact, approximately 20-min passes from the initial enhancement of $1-2-\mathrm{km} \zeta$ to the rapid intensification of the surface vortex. Assuming that the model is accurately representing real within-storm processes, this suggests potential for lead time during real-time warning operations of these events, provided radar coverage is sufficient to observe such trends in low to midlevel rotation. The key features noted above must be spatially collocated for the chain of processes to succeed. The processes also appear to be self-limiting, as eventually, downward-directed ACCDNL resulting from the 


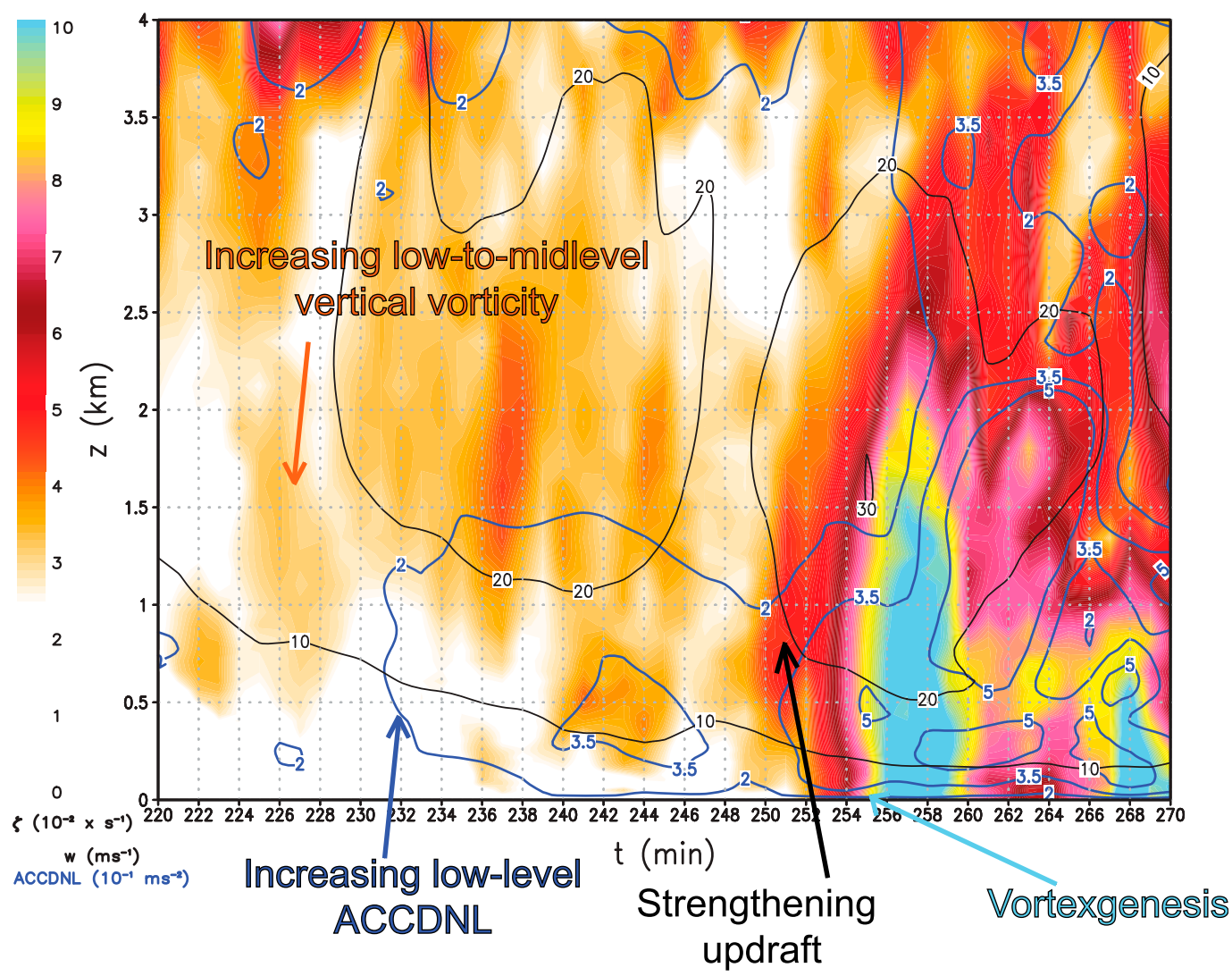

FIG. 17. Vertical time series of maximum $\zeta\left(\times 10^{-2} \mathrm{~s}^{-1}\right.$; shading), $w\left(\mathrm{~m} \mathrm{~s}^{-1}\right.$, black contours), and ACCDNL $\left(\times 10 \mathrm{~m} \mathrm{~s}^{-2}\right.$, blue contours) within a $10 \mathrm{~km}$ by $10 \mathrm{~km}$ box surrounding the strongest updraft, leading to the development of the strongest vortices in the control simulation.

strengthening surface vortex disrupts the updraft and limits further intensification.

Potential failure points in the series of processes leading to vortexgenesis will be examined in the following section across the varying environmental base states. It is suspected that each environment will produce surface $\zeta$ that could potentially be converged and stretched. This follows the control simulation detailed above, which showed plentiful $\zeta$ within the cold pool, and Coffer and Parker (2017), who posited that this final step in the process of tornadogenesis was nearly entirely dependent on above-ground storm characteristics. It is hypothesized that weaker low-level lapse rates may struggle to support initially strong low-level updrafts given extremely modest instability in the lowest $3 \mathrm{~km}$ above ground (Hampshire et al. 2017). In this case, the initial updraft may simply be too weak to support the development of a strong surface vortex. Within weaker low-level shear environments, it is suspected that the production of low- to midlevel $\zeta$ will be limited, given the documented sensitivity of low-level $\zeta$ production to low-level shear in both supercells and QLCSs. This could preclude the strengthening of a low-level updraft that precedes vortexgenesis. We evaluate the validity of these hypotheses via the various sensitivity simulations in the following section.

\section{c. Sensitivity tests}

Convective mode generally differs little across the matrix of five simulations (cf. Figs. 4 and 18), even as the areal coverage of convection varies somewhat. Differences in storm motion can be noted early in the comparisons, when some convection (e.g., -01s) remains closer to the initiating boundary (e.g., located at approximately $x=-40 \mathrm{~km}$ in Fig. 18d). Additionally, Hovmöller plots reveal considerable differences in rotational characteristics between the runs (Fig. 19). In particular, it appears that 01s plays a substantial role in both the number of incipient surface vortices that develop and the strength and longevity of rotating updrafts. In -01 s, the number of surface vortices decreases greatly, while the opposite is true in $+01 \mathrm{~s}$ (cf. Figs. 19a,b). Additionally, $+01 \mathrm{~s}$ supports a long-lived rotating updraft, while rotating 

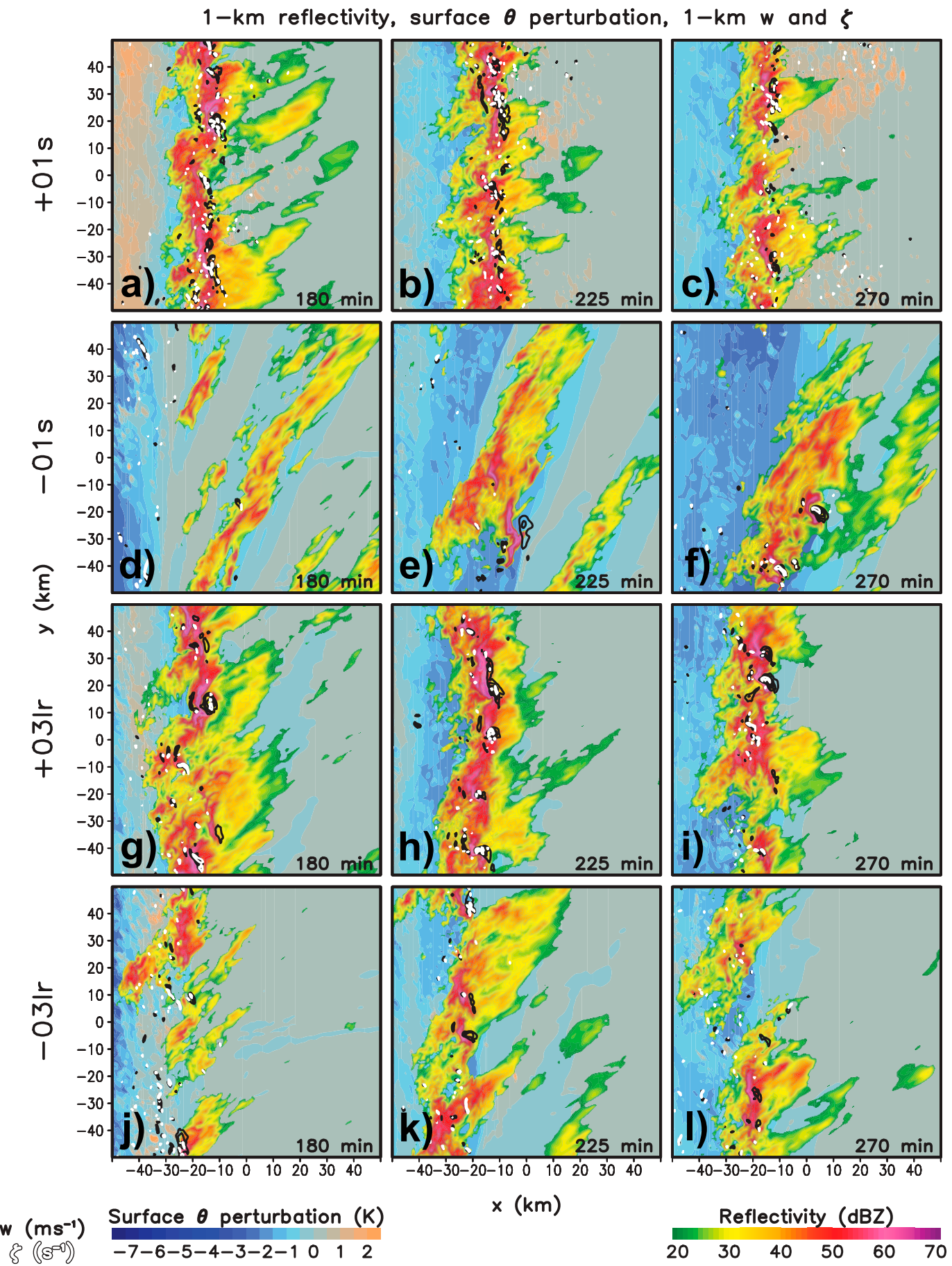

FIG. 18. As in Fig. 4, but for (a)-(c) +01s, (d)-(f) -01s, (g)-(i) +031r, and (j)-(l) $-031 \mathrm{l}$ for (from left to right) $t=180,225$, and $270 \mathrm{~min}$.

updrafts in -01 s tend to be transient compared to the control simulation or +01 s. Similarly, within -031 , rotating updrafts are fairly weak and short lived, whereas the strongest updrafts and surface vortices across the matrix of simulations were observed in +031 r (cf. Figs. 19c,d). The most prominent sensitivities in the lapse rate tests appear to be associated with the number of strong, rotating updrafts and the typical strength of surface vortices; both of these decrease as 031r decreases.

Results from the tracking algorithms generally corroborate these findings (Fig. 20). Compared to the control simulation, the number, strength, and longevity 

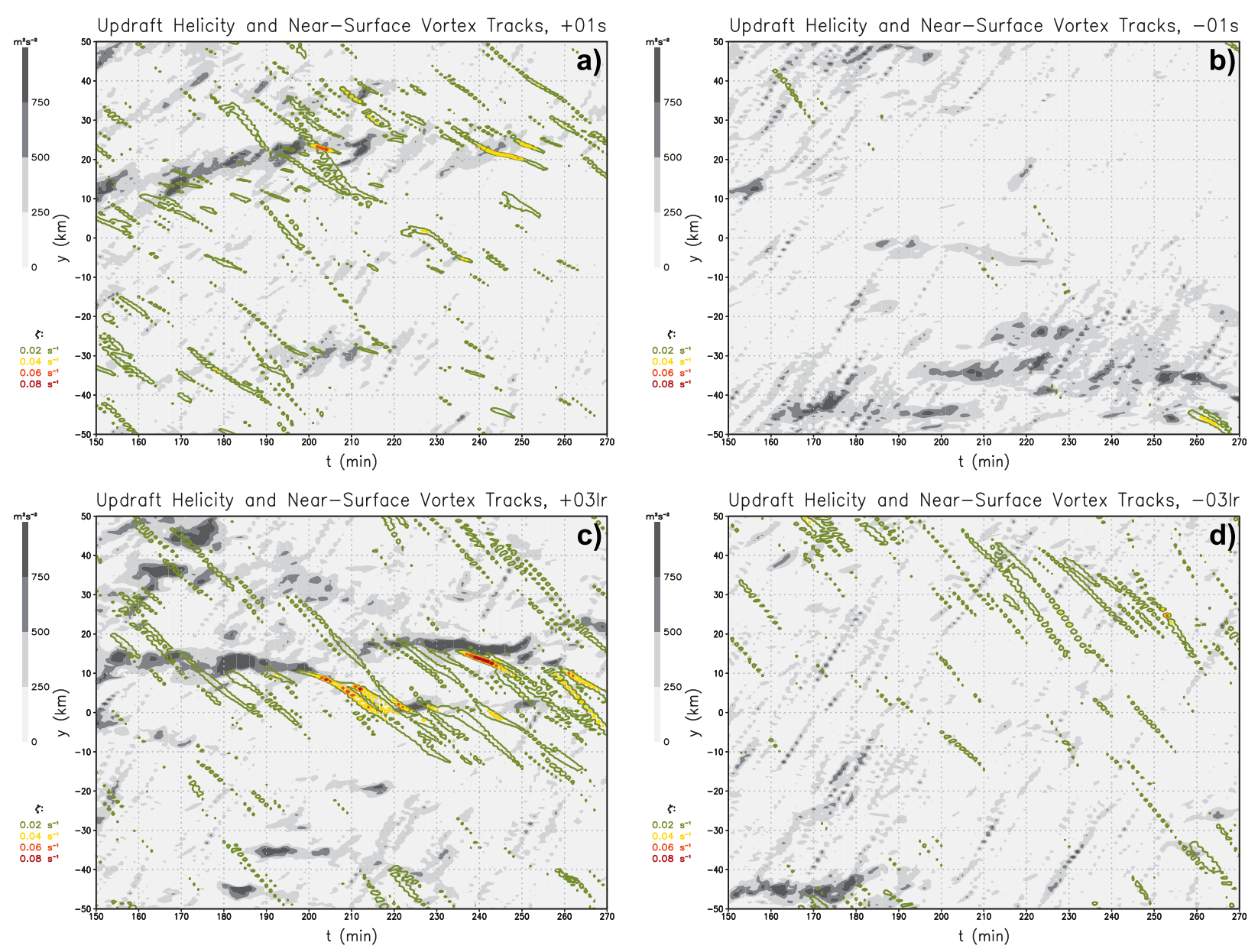

FIG. 19. As in Fig. 5, but for (a) +01s, (b)

$-01 \mathrm{~s},(\mathrm{c})+031 \mathrm{l}$, and (d) $-031 \mathrm{r}$ simulations.

of updrafts and low-level vortices decrease within the upper half of the distributions in $-031 \mathrm{r}$ and $-01 \mathrm{~s}$, while they generally increase or are comparable to the control run for $+031 \mathrm{r}$ and $+01 \mathrm{~s}$. However, there is substantial overlap in these distributions, suggesting that the biggest practical difference is in the number of the aforementioned features. Additionally, as either 01s or 031r increases, the distributions of ACCD and ACCB within long-lived (i.e., duration $\geq 20 \mathrm{~min}$ ) updrafts shift toward higher values (Fig. 21). Notably, ACCD is almost always larger, and tends to vary more, than ACCB within these updrafts, regardless of the associated environment, as was documented in the control run. This confirms that the main change in forcing among differing base-state environments is dynamic in nature, underscoring the importance of embedded rotating updrafts. To further compare the simulations in their entirety, we use plots of exceedance frequency (i.e., the number of times a given threshold value of a field was met or exceeded over the course of each simulation). Here, we use thresholds of $0.03 \mathrm{~s}^{-1}, 0.3 \mathrm{~m} \mathrm{~s}^{-2}$, and $20 \mathrm{~m} \mathrm{~s}^{-1}$ for $\zeta$, ACCDNL, and $w$ (Fig. 22), respectively. Given that the processes of utmost importance appeared to be limited to low levels, we focus our analysis on the layer from the surface to $1.5 \mathrm{~km}$ AGL.

Low-level $\zeta$ appears to be largely dependent on the magnitude of $01 \mathrm{~s}$, with approximately an order of magnitude increase in the number of occurrences of $\zeta$ of at least $0.03 \mathrm{~s}^{-1}$ across the lowest $1 \mathrm{~km}$ in $+01 \mathrm{~s}$ compared to $-01 \mathrm{~s}$ (Fig. 22a). This gives $+01 \mathrm{~s}$ a considerable advantage over $-01 \mathrm{~s}$ in the chain of processes leading to surface vortexgenesis and contributes to increased ACCDNL and, accordingly, $w$ (Figs. 22b,c) in the lowest $1 \mathrm{~km}$. Although prior QLCS studies have shown that longer-lived mesovortices are more likely to produce surface vortices (e.g., Przybylinski et al. 2000; Atkins et al. 2004, 2005), it is not entirely clear how our simulated embedded supercells correspond to the observed convection in their studies; plausibly, the formation mechanisms and characteristics could be rather similar, particularly given the differing resolution and techniques used in these analyses. 

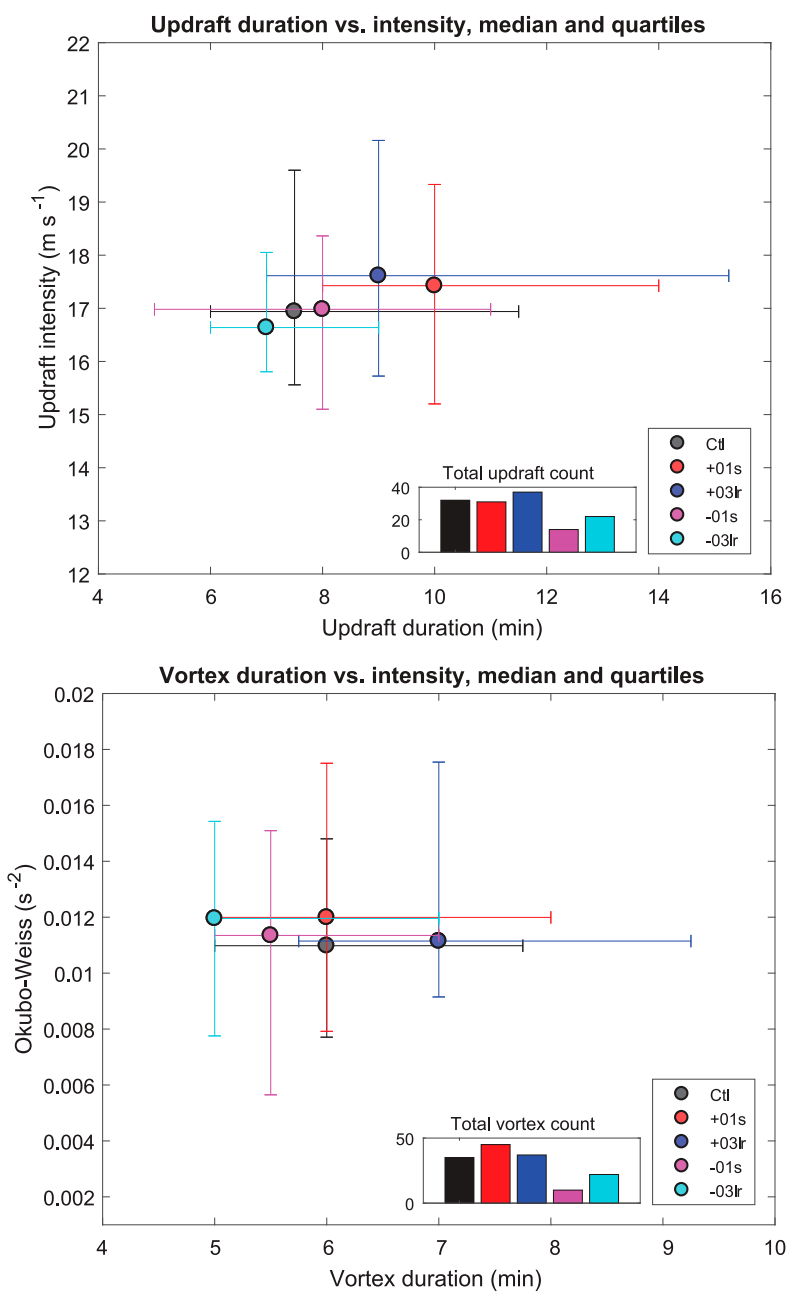

FIG. 20. Scatterplot of (top) mean updraft duration (min) against mean maximum updraft intensity $\left(\mathrm{m} \mathrm{s}^{-1}\right)$ and (bottom) mean vortex duration ( $\mathrm{min}$ ) against mean maximum vortex intensity (Okubo-Weiss parameter, $\mathrm{s}^{-2}$ ) for the tracked features in each simulation. Error bars extend to the 25th and 75th percentiles of each distribution.

The primary difference between $+031 \mathrm{r}$ and $-031 \mathrm{r}$ lies in the $w$, where an order of magnitude increase is again noted in $+031 \mathrm{r}$ throughout most of the lowest $1.5 \mathrm{~km}$ when compared to -031 r (Fig. 22c). Comparable differences are noted in ACCDNL, while exceedance frequencies for $\zeta$ are a bit more similar (Figs. 22a,b). Interestingly, the $+01 \mathrm{~s} \zeta$ exceedance frequencies outpace those in $+031 \mathrm{r}$, while $+031 \mathrm{r}$ has larger exceedance frequencies at most heights for ACCDNL; this is likely because +01 s has more low-level vortices, but those that form in +031 tend to be stronger and deeper. Altogether, this analysis supports the contention that steeper 031r support stronger low-level updrafts, while 01s affects the number and strength of embedded rotation centers within the updrafts. The ideal HSLC environment for the development of severe weather
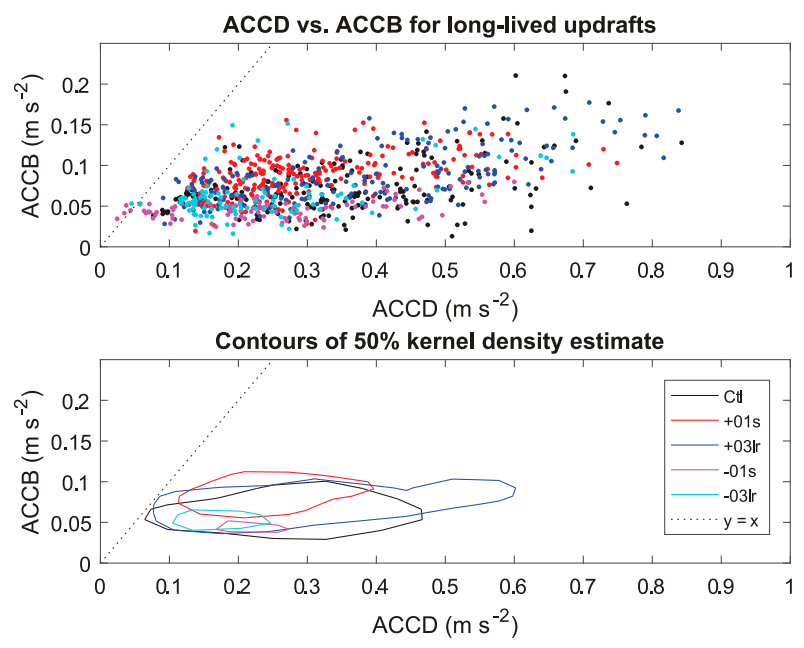

FIG. 21. (top) Scatterplot of ACCD and ACCB for each minute of tracked long-lived updrafts within the respective simulations. (bottom) A kernel density estimate encompassing $50 \%$ of the total distribution, extending from the densest bin.

would exhibit both steep low-level lapse rates and large low-level shear vector magnitude in order to benefit from both sensitivities, as identified in prior studies (Sherburn and Parker 2014; Sherburn et al. 2016). With these broad differences in the distributions in mind, we can shift our analysis to the strongest updraft in each case to investigate the relevant processes in more detail.

Within the strongest updraft of -01s, low-level ACCD is considerably weaker than in the control simulation. This can be attributed primarily to ACCDNL (Fig. 23), which is limited due to a lack of low-level $\zeta$. Thus, as suggested above, the failure point in -01 s appears to be a lack of sufficient dynamic forcing for ascent due to decreased low- to midlevel $\zeta$. This limits the strength of low-level updrafts and precludes the convergence and stretching of surface $\zeta$, which is generally present once a cold pool is established in each primary matrix simulations. Therefore, there is likely a minimum value of $01 \mathrm{~s}$ necessary for the development of strong, long-lived, lowlevel vortices capable of supporting tornadoes within HSLC environments.

As $01 \mathrm{~s}$ increases beyond the control value, the response is not entirely straightforward. The number of surface vortices indeed increases in +01 s (as shown in Fig. 20), but embedded rotating updrafts become less organized. As 01s increases, the environmental Richardson number within this idealized framework falls well below the critical value of 0.25 , leading to the development of widespread turbulence. Within +01 s, this does not disrupt the convection to a point where surface vortices are precluded. Low-level ACCD fields associated with the strongest updraft (an embedded supercell) remain 

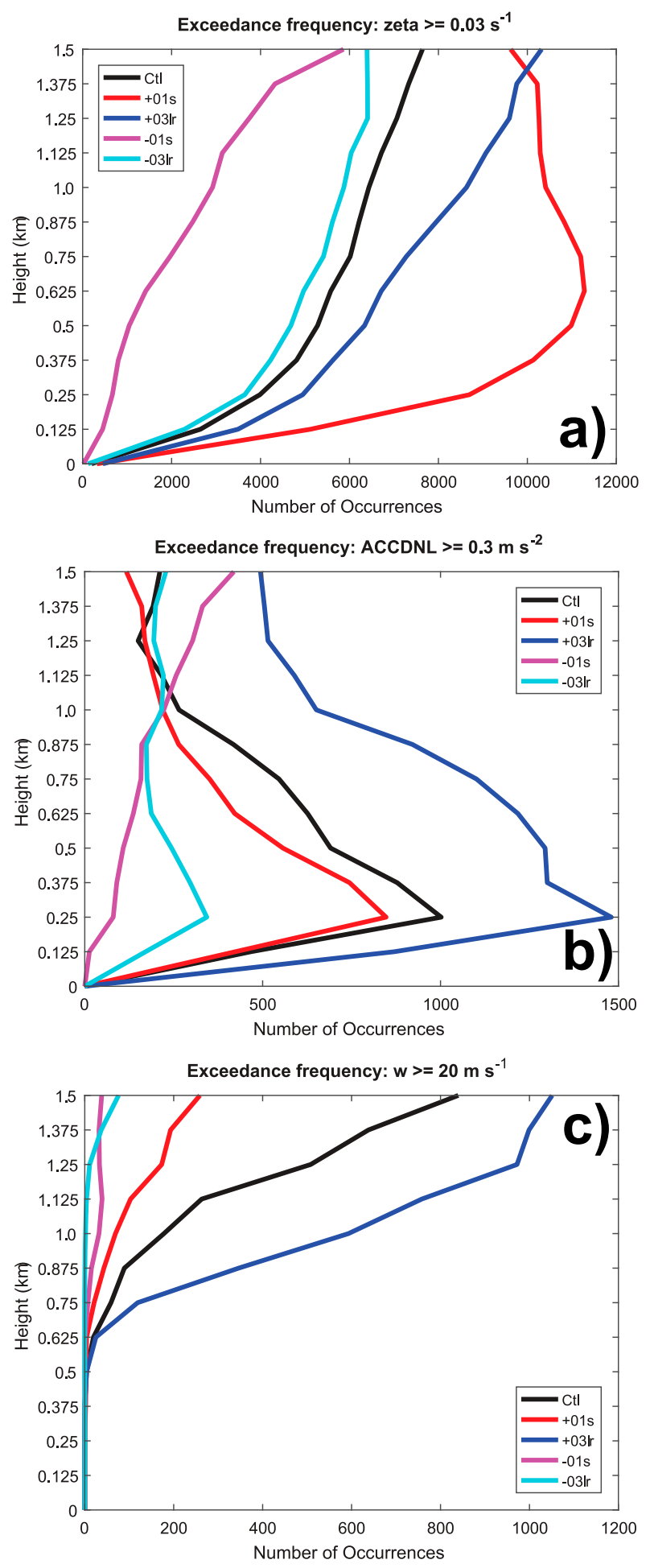

FIG. 22. Exceedance frequency for (a) $\zeta$ of $0.03 \mathrm{~s}^{-1}$, (b) ACCDNL of $0.3 \mathrm{~m} \mathrm{~s}^{-2}$, and (c) $w$ of $20 \mathrm{~m} \mathrm{~s}^{-1}$ for all simulations. sufficient to produce appreciable updrafts in the lowest $1 \mathrm{~km}$, facilitating the development of an intense surface vortex, albeit one that is weaker and shallower than in the control simulation (Fig. 24). However, in a supplemental simulation with $01 \mathrm{~s}$ of $50 \mathrm{kt}$ (i.e., $10 \mathrm{kt}$ stronger than in $+01 \mathrm{~s})$, tracked updrafts become shorter lived and weaker on the mean (not shown). Thus, in addition to the minimum threshold of $01 \mathrm{~s}$ for the development of severe hazards in HSLC environments, it is possible that there is a threshold beyond which increasing $01 \mathrm{~s}$ no longer improves the potential for the production of severe hazards. This possibility is somewhat supported by prior environmental studies that show a broader range of low-level shear vector magnitude values for warned but nonsevere HSLC convection when compared to severe HSLC convection (e.g., Sherburn et al. 2016). It may also be that, in such strongly sheared environments with low Richardson numbers, a fully turbulent representation of the atmospheric boundary layer is essential in order to acquire a statistically steady ambient environment.

Clearly, steepening 031r leads to an increase in the severity of HSLC convection, given the environment's ability to produce stronger and longer-lived low-level updrafts and vortices (Fig. 20). This is supported by a vertical time series of the primary rotating updraft (Fig. 25), which exhibits a rapid increase in low-level $\zeta$ and the subsequent development of an intense surface vortex. The initial strength of updrafts in -031 r tends to be comparatively weaker (e.g., Figs. 20 and 21), hindering the remaining processes in their ability to produce a strong surface vortex. Generally, weaker updrafts early in the $-031 \mathrm{r}$ convection's lifetime can likely be attributed to less buoyancy in -031r compared to the control environment (Fig. 21); however, ACCD is weaker in -031 , as well.

Across the five preceding simulations, the most intense vortices tend to be affiliated with the strongest, rotating updrafts. The results suggest that decreasing either $031 \mathrm{r}$ or $01 \mathrm{~s}$ decreases the potential for stronger, longer-lived, and more numerous low-level updrafts and surface vortices. These sensitivities arise through differences in both initial updraft strengths and feedbacks associated with ACCDNL that develops beneath rotation aloft. Though many vortices and updrafts within the distributions are weak and transient, they are important to consider because their origin locations and mechanisms are generally similar to those features that become dominant. Increased numbers of low-level vortices, for example, raise the potential that one of these vortices will benefit from favorable collocation with an overlying updraft or associated near-surface convergence zone, thus leading to rapid intensification. Meanwhile, stronger 

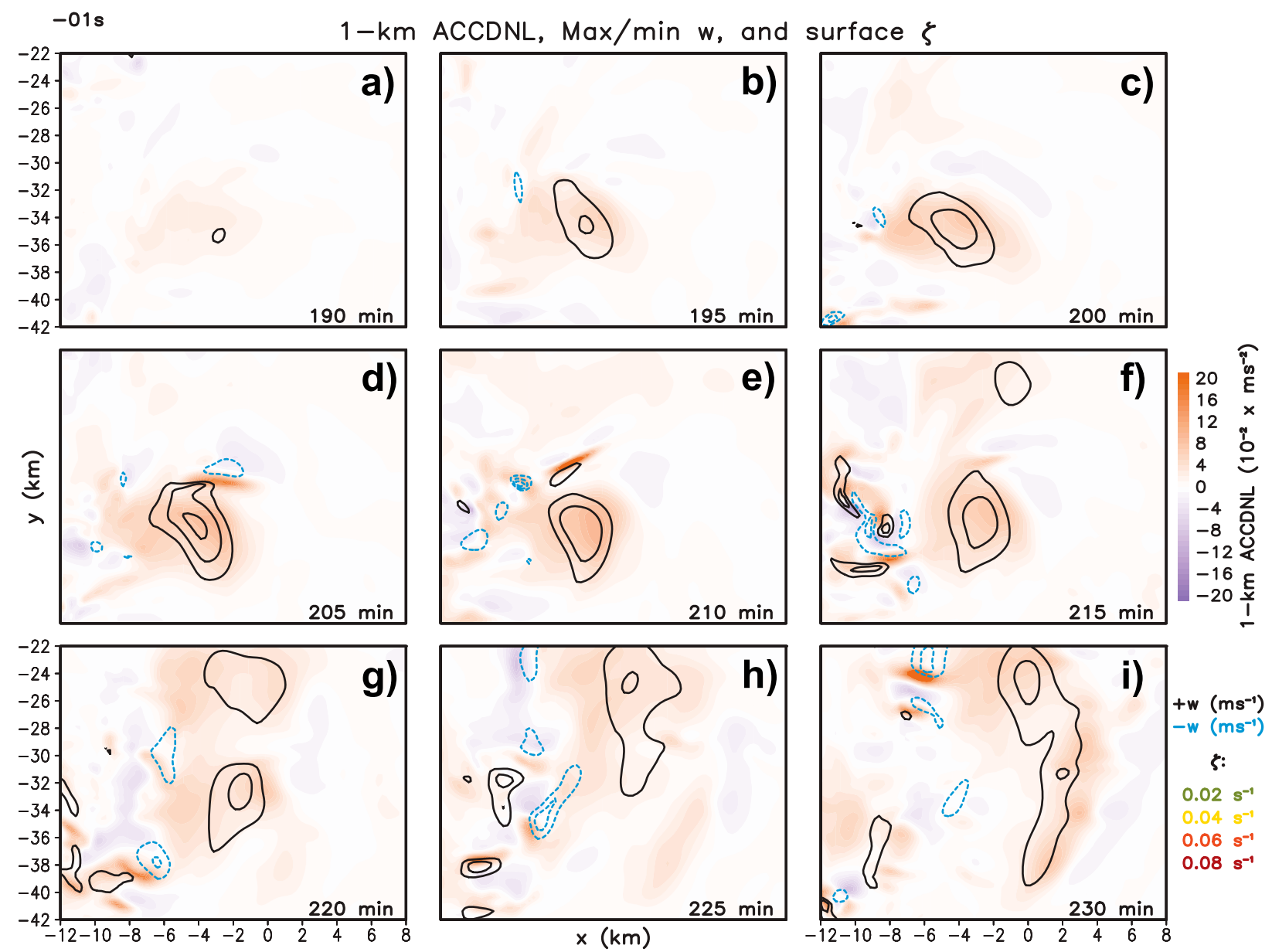

FIG. 23. As in Fig. 15, but for -01 s. low-level updrafts would favor more rapid stretching of near-surface vorticity, again promoting intensification of low-level vortices. For HSLC supercells embedded within a broader QLCS, these basic sensitivities help explain the parameters identified as the most skillful in the environmental studies of Sherburn and Parker (2014) and Sherburn et al. (2016), who found that the potential for tornadoes increased statistically with steepening lowlevel lapse rates and increased low-level shear vector magnitudes. In addition to within-storm processes, modified lapse rates and shear vector magnitudes also impact the convective system's initiation and cold pool evolution. We envision exploring these additional interactions between the convective system and ambient environment within less idealized experimental designs in the future.

\section{Summary and conclusions}

Investigations into severe and nonsevere HSLC convection have identified key discriminators between their environments. In particular, low-level lapse rates and shear vector magnitudes-along with the strength of synoptic scale forcing for ascent-have been shown to differentiate well between those HSLC environments supporting severe convection and those that are limited to nonsevere convection (Sherburn and Parker 2014; Sherburn et al. 2016; King et al. 2017). The idealized simulations herein have identified physical explanations behind these sensitivities associated with quasi-supercellular structures within a broader QLCS. Additionally, they have provided an overview of the processes leading to the development of strong surface vortices in HSLC environments.

Given a sufficiently strong supercellular updraft in an HSLC environment, the following processes may lead to the development of a strong surface vortex:

1) Low- to midlevel $\zeta$ (between 0.5 and $2 \mathrm{~km}$ AGL) increases as the midlevel updraft develops and intensifies;

2) in response to the increase in low- to midlevel $\zeta$, upward-pointing accelerations via ACCDNL increase 


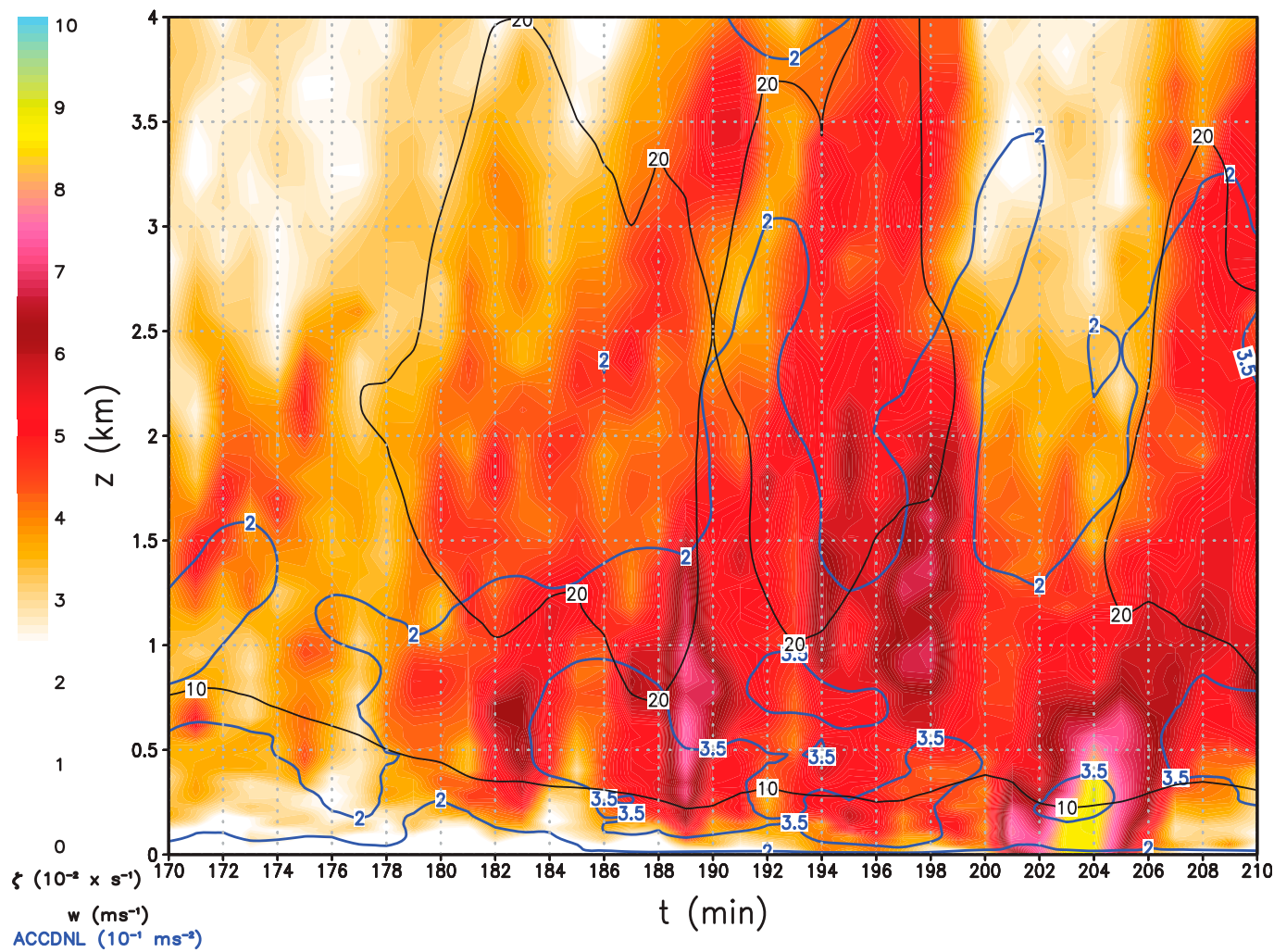

FIG. 24. As in Fig. 17, but for +01 s.

in low levels, which is particularly important in these low-CAPE environments;

3) due to increased accelerations in low levels, low-level $w$ intensifies; and

4) increased low-level $w$ provides increased potential for tilting and especially stretching of surface vorticity, particularly in the lowest $500 \mathrm{~m}$ above ground. This facilitates the development of a strong surface vortex.

Notably, this final step will only succeed if there is nontrivial $\zeta$ at the ground and this $\zeta$ is spatially collocated with the overlying rotating updraft. Following the identification of these processes in a control simulation, sensitivity tests were used to determine how these processes were affected as either $01 \mathrm{~s}$ or $031 \mathrm{l}$ decreased or increased.

Although the particular matrix of simulations conducted here investigated only a small portion of the HSLC parameter space, the values for $01 \mathrm{~s}$ and $031 \mathrm{r}$ tested represent a realistic sampling of environments near the most discriminatory values of these fields according to Sherburn and Parker (2014) and Sherburn et al. (2016). Thus, we are confident in the following summary of sensitivities with regard to embedded supercellular convection within HSLC environments:
- The primary impact of decreasing $01 \mathrm{~s}$ is decreasing the production of low- to midlevel $\zeta$ via tilting by the developing updrafts. This limits low-level ACCDNL and, thus, low-level $w$.

- The primary impact of decreasing 03lr is decreasing the initial strength of updrafts due to generally weaker buoyant accelerations. This limits the potential for tilting and stretching of vorticity in low levels.

- Decreasing either 01s or 031r limits the potential for severe weather in HSLC environments. Either situation prevents the chain of processes extending from the development of a strong updraft to the eventual development of a strong surface vortex from being successful.

Regardless of environment, ACCD, rather than ACCB, were dominant in forcing updrafts. This is no surprise given that the key updrafts here are near or below the level of free convection (LFC), where ACCB will be minimal. These findings corroborate prior research indicating that ACCD dominated in both convection with rotating updrafts and in HSLC environments (e.g., Weisman and Klemp 1984; McCaul and Weisman 1996; Weisman and Rotunno 2000; Coffer and Parker 2017). This implies that HSLC environments incapable of producing sufficient low- to midlevel $\zeta$-that is, those 


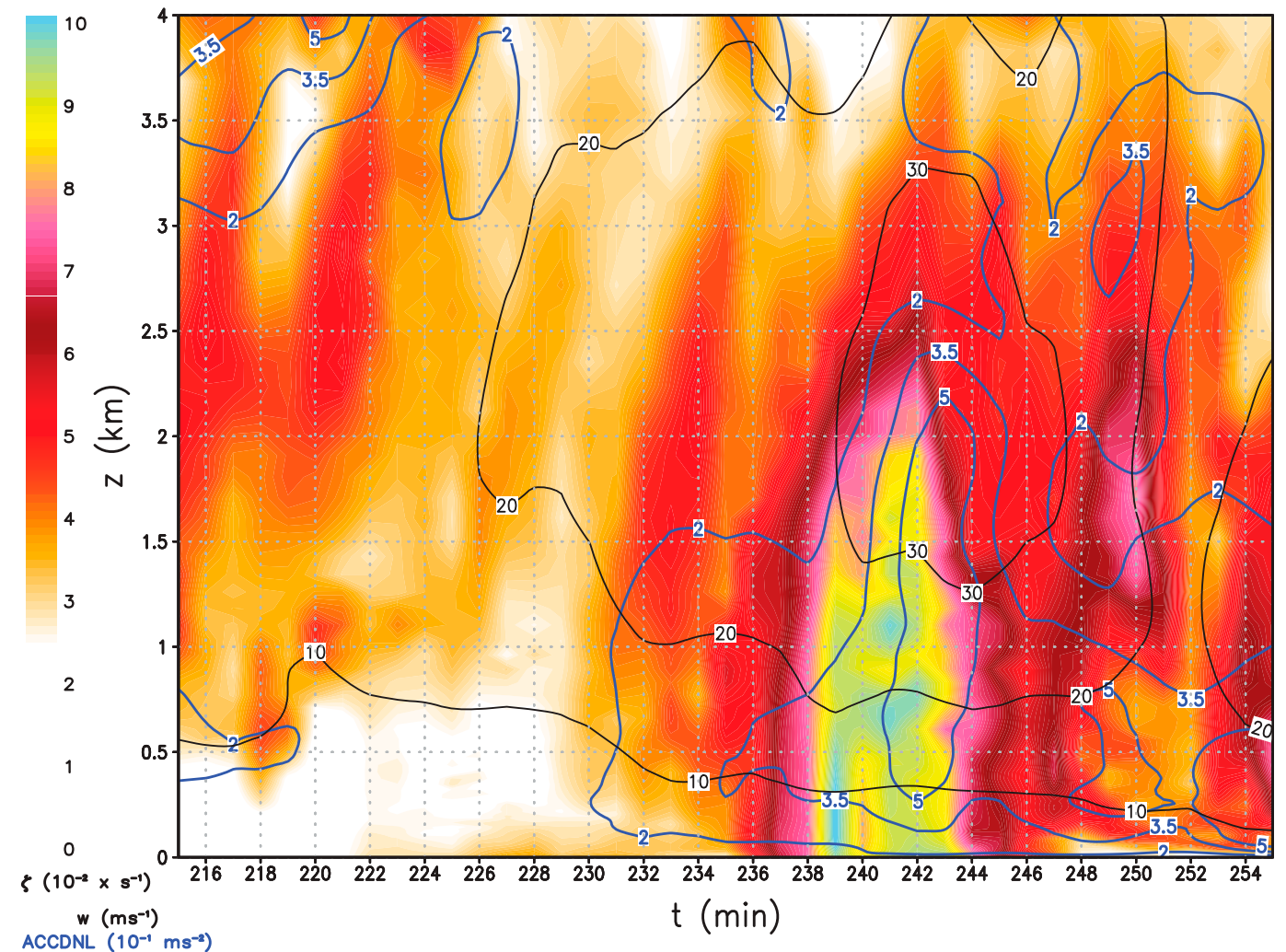

FIG. 25. As in Fig. 17, but for +031 .

with meager 01s-will have a much lower potential of producing strong surface vortices, as found here. Furthermore, it also suggests that the potential for the development of a strong surface vortex could be evaluated to some degree in real-time with radial velocity products, assuming sufficient radar coverage. Indeed, in the simulations presented here, nearly $20 \mathrm{~min}$ of time passed between the initial intensification of midlevel $(1-2 \mathrm{~km}) \zeta$ and the development of the surface vortex.

There is no shortage of additional work on HSLC environments to be undertaken. Future work should continue to test the sensitivities of HSLC convection to additional environmental characteristics, such as midlevel shear vector magnitudes (also shown by Sherburn and Parker 2014 to be skillful discriminators), hodograph shapes and orientations, forcing mechanisms and strengths, and the orientation and strength of nearby boundaries. Further work at higher resolutions, where low-level and surface vortices are better resolved, would be especially useful in analyzing sources of vorticity and how HSLC vortices differ from those in higher-CAPE convection. Given that many of the processes preceding surface vortexgenesis occurred very close to the lowest model level, future work should employ a more realistic bottom boundary condition to determine the impact of drag on these processes provided that suitable parameterizations can be developed for idealized HSLC convection simulations. Finally, studies comparing observational data and model output, particularly with tools such as a radar emulator, would be helpful in identifying precursors to lowlevel and surface vortexgenesis or the onset of damaging straight-line winds, which could improve detection and lead time of severe hazards in real cases. Additionally, it is important to determine how many HSLC QLCS tornadoes occur in association with embedded quasi-supercellular structures, such as those observed in our simulations. However, we acknowledge that many HSLC QLCS tornadoes are likely associated with bow-echo type structures and mesovortices, which likely differ physically from the supercellular mechanisms discussed herein. In spite of the lingering questions and abundant potential future work, the simulations herein provide insight into why two of the most skillful environmental parameters, low-level shear vector magnitudes and lapse rates, discriminate well between severe and nonsevere HSLC convection.

Acknowledgments. We would like to acknowledge collaborators in the Collaborative Science, Technology, and Applied Research (CSTAR) program from regional 
and national National Weather Service offices and centers, along with those at NC State University, for their contributions to this work. Additionally, we thank Drs. DelWayne Bohnenstiehl, Gary Lackmann, and Sandra Yuter, who provided constructive criticism for this work as members of KS's Ph.D. committee. We greatly appreciate Dr. George Bryan's continuous dedication to the development and improvement of CM1, which was used in this study. Additionally, we would like to acknowledge the developers of the Sounding/Hodograph Analysis and Research Program in Python (SHARPpy; Blumberg et al. 2017), which was used for analysis and image production in this study. We also acknowledge three anonymous reviewers and Dr. Matthew Bunkers, whose comments have led to a greatly improved manuscript. This research was funded by NSF Grant AGS-1156123 and NOAA Grants NA14NWS4680013, NA15OAR4590235, and NA16OAR4590213.

\section{REFERENCES}

Adlerman, E. J., and K. K. Droegemeier, 2005: The dependence of numerically simulated cyclic mesocyclogenesis upon environmental vertical wind shear. Mon. Wea. Rev., 133, 35953623, https://doi.org/10.1175/MWR3039.1.

Anderson-Frey, A. K., Y. P. Richardson, A. R. Dean, R. L. Thompson, and B. T. Smith, 2016: Investigation of near-storm environments for tornado events and warnings. Wea. Forecasting, 31, 1771-1790, https://doi.org/10.1175/WAF-D-16-0046.1.

Atkins, N. T., and M. St. Laurent, 2009a: Bow echo mesovortices. Part I: Processes that influence their damaging potential. Mon. Wea. Rev., 137, 1497-1513, https://doi.org/10.1175/2008MWR2649.1.

— , and - 2009b: Bow echo mesovortices. Part II: Their genesis. Mon. Wea. Rev., 137, 1514-1532, https://doi.org/ 10.1175/2008MWR2650.1.

_ J. M. Arnott, R. W. Przybylinski, R. A. Wolf, and B. D. Ketcham, 2004: Vortex structure and evolution within bow echoes. Part I: Single-Doppler and damage analysis of the 29 June 1998 derecho. Mon. Wea. Rev., 132, 2224-2242, https://doi.org/ 10.1175/1520-0493(2004)132<2224:VSAEWB > 2.0.CO;2.

—, C. S. Bouchard, R. W. Przybylinski, R. J. Trapp, and G. Schmocker, 2005: Damaging surface wind mechanisms within the 10 June 2003 Saint Louis bow echo during BAMEX. Mon. Wea. Rev., 133, 2275-2296, https://doi.org/10.1175/ MWR2973.1.

Blumberg, W. G., K. T. Halbert, T. A. Supinie, P. T. Marsh, R. L. Thompson, and J. A. Hart, 2017: SHARPpy: An open source sounding analysis toolkit for the atmospheric sciences. Bull. Amer. Meteor. Soc., 98, 1625-1636, https://doi.org/10.1175/ BAMS-D-15-00309.1.

Brooks, H. E., J. W. Lee, and J. P. Craven, 2003: The spatial distribution of severe thunderstorm and tornado environments from global reanalysis data. Atmos. Res., 67-68, 73-94, https:// doi.org/10.1016/S0169-8095(03)00045-0.

Bryan, G. H., and J. M. Fritsch, 2002: A benchmark simulation for moist nonhydrostatic numerical models. Mon. Wea. Rev., 130, 2917-2928, https://doi.org/10.1175/1520-0493(2002)130<2917: ABSFMN $>2.0 . \mathrm{CO} ; 2$.
_- and R. Rotunno, 2009: Evaluation of an analytical model for the maximum intensity of tropical cyclones. J. Atmos. Sci., 66, 3042-3060, https://doi.org/10.1175/2009JAS3038.1.

Bunkers, M. J., D. A. Barber, R. L. Thompson, R. Edwards, and J. Garner, 2014: Choosing a universal mean wind for supercell motion prediction. J. Oper. Meteor., 2, 115-129, https://doi.org/ 10.15191/nwajom.2014.0211.

Coffer, B. E., and M. D. Parker, 2015: Impacts of increasing lowlevel shear on supercells during the early evening transition. Mon. Wea. Rev., 143, 1945-1969, https://doi.org/10.1175/ MWR-D-14-00328.1.

$\longrightarrow$, and — 2017: Simulated supercells in nontornadic and tornadic VORTEX2 environments. Mon. Wea. Rev., 145, 149180, https://doi.org/10.1175/MWR-D-16-0226.1.

,-- J. M. L. Dahl, L. J. Wicker, and A. J. Clark, 2017: Volatility of tornadogenesis: An ensemble of simulated nontornadic and tornadic supercells in VORTEX2 environments. Mon. Wea. Rev., 145, 4605-4625, https://doi.org/10.1175/ MWR-D-17-0152.1.

Cope, A. M., 2004: An early morning mid-Atlantic severe weather episode: Short-lived tornadoes in a high-shear low-instability environment. 22nd Conf. on Severe Local Storms, Hyannis, MA, Amer. Meteor. Soc., P1.4, https://ams.confex.com/ams/ 11aram22sls/webprogram/Paper81834.html.

Dahl, J. M. L., M. D. Parker, and L. J. Wicker, 2014: Imported and storm-generated near-ground vertical vorticity in a simulated supercell. J. Atmos. Sci., 71, 3027-3051, https://doi.org/10.1175/ JAS-D-13-0123.1.

Davenport, C. E., and M. D. Parker, 2015: Impact of environmental heterogeneity on the dynamics of a dissipating supercell thunderstorm. Mon. Wea. Rev., 143, 4244-4277, https://doi.org/ 10.1175/MWR-D-15-0072.1.

Davies-Jones, R., 2002: Linear and nonlinear propagation of supercell storms. J. Atmos. Sci., 59, 3178-3205, https://doi.org/ 10.1175/1520-0469(2003)059<3178:LANPOS >2.0.CO;2.

- 2015: A review of supercell and tornado dynamics. Atmos. Res., 158-159, 274-291, https://doi.org/10.1016/j.atmosres.2014.04.007. , and H. Brooks, 1993: Mesocyclogenesis from a theoretical perspective. The Tornado: Its Structure, Dynamics, Prediction, and Hazards, C. Church et al., Eds., Amer. Geophys. Union, 105-114.

Davis, J. M., and M. D. Parker, 2014: Radar climatology of tornadic and nontornadic vortices in high-shear, low-CAPE environments in the mid-Atlantic and southeastern United States. Wea. Forecasting, 29, 828-853, https://doi.org/10.1175/ WAF-D-13-00127.1.

Dawson, D. T., M. Xue, A. Shapiro, J. A. Milbrandt, and A. D. Schenkman, 2016: Sensitivity of real-data simulations of the 3 May 1999 Oklahoma City tornadic supercell and associated tornadoes to multimoment microphysics. Part II: Analysis of buoyancy and dynamic pressure forces in simulated tornadolike vortices. J. Atmos. Sci., 73, 1039-1061, https://doi.org/ 10.1175/JAS-D-15-0114.1.

Dean, A. R., and R. S. Schneider, 2012: An examination of tornado environments, events, and impacts from 2003-2012. 26th Conf. on Severe Local Storms, Nashville, TN, Amer. Meteor. Soc., 60, https://ams.confex.com/ams/26SLS/webprogram/ Paper211580.html.

Esterheld, J. M., and D. J. Giuliano, 2008: Discriminating between tornadic and non-tornadic supercells: A new hodograph technique. Electron. J. Severe Storms Meteor., 3 (2), http://www.ejssm.org/ojs/index.php/ejssm/article/ viewArticle/33. 
Godfrey, E. S., R. J. Trapp, and H. E. Brooks, 2004: A study of the pre-storm environment of tornadic quasi-linear convective systems. 22nd Conf. on Severe Local Storms, Hyannis, MA, Amer. Meteor. Soc., 3A.5, https://ams.confex.com/ams/ 11 aram22sls/techprogram/paper_81388.htm.

Hampshire, N. L., R. M. Mosier, T. M. Ryan, and D. E. Cavanaugh, 2017: Relationship of low-level instability and tornado damage rating based on observed soundings. J. Oper. Meteor., 6, 1-12, https://doi.org/10.15191/nwajom.2018.0601.

King, J. R., M. D. Parker, K. D. Sherburn, and G. M. Lackmann, 2017: Rapid evolution of cool season, low CAPE severe thunderstorm environments. Wea. Forecasting, 32, 763-779, https://doi.org/10.1175/WAF-D-16-0141.1.

Klemp, J. B., 1987: Dynamics of tornadic thunderstorms. Annu. Rev. Fluid Mech., 19, 369-402, https://doi.org/10.1146/ annurev.fl.19.010187.002101.

Leslie, L. M., and R. K. Smith, 1978: The effect of vertical stability on tornadogenesis. J. Atmos. Sci., 35, 1281-1288, https://doi.org/ 10.1175/1520-0469(1978)035<1281:TEOVSO > 2.0.CO;2.

Mansell, E. R., C. L. Ziegler, and E. C. Bruning, 2010: Simulated electrification of a small thunderstorm with two-moment bulk microphysics. J. Atmos. Sci., 67, 171-194, https://doi.org/ 10.1175/2009JAS2965.1.

Markowski, P. M., 2016: An idealized numerical simulation investigation of the effects of surface drag on the development of near-surface vertical vorticity in supercell thunderstorms. J. Atmos. Sci., 73, 4349-4385, https://doi.org/10.1175/JAS-D16-0150.1.

—_ 2018: A review of the various treatments of the surface momentum flux in severe storms simulations: Assumptions, deficiencies, and alternatives. 29th Conf. on Severe Local Storms, Stowe, VT, Amer. Meteor. Soc., 7.3, https://ams.confex.com/ ams/29SLS/webprogram/Paper348116.html.

— potential problem for convective storm simulations. Mon. Wea. Rev., 144, 1841-1850, https://doi.org/10.1175/MWR-D15-0439.1.

_ , and Y. P. Richardson, 2014: The influence of environmental low-level shear and cold pools on tornadogenesis: Insights from idealized simulations. J. Atmos. Sci., 71, 243-275, https:// doi.org/10.1175/JAS-D-13-0159.1.

— , and — 2017: Large sensitivity of near-surface vertical vorticity development to heat sink location in idealized simulations of supercell-like storms. J. Atmos. Sci., 74, 1095-1104, https://doi.org/10.1175/JAS-D-16-0372.1.

McCaul, E. W., Jr., and M. L. Weisman, 1996: Simulation of shallow supercell storms in landfalling hurricane environments. Mon. Wea. Rev., 124, 408-429, https://doi.org/10.1175/15200493(1996)124<0408:SOSSSI >2.0.CO;2.

Okubo, A., 1970: Horizontal dispersion of floatable particles in the vicinity of velocity singularities such as convergences. DeepSea Res. Oceanogr. Abstr., 17, 445-454.

Orf, L., R. Wilhelmson, B. Lee, C. Finley, and A. Houston, 2017: Evolution of a long-track violent tornado within a simulated supercell. Bull. Amer. Meteor. Soc., 98, 45-68, https://doi.org/ 10.1175/BAMS-D-15-00073.1.

Parker, M. D., 2007: Simulated convective lines with parallel stratiform precipitation. Part II: Governing dynamics and associated sensitivities. J. Atmos. Sci., 64, 289-313, https:// doi.org/10.1175/JAS3854.1.

_ 2010 : Relationship between system slope and updraft intensity in squall lines. Mon. Wea. Rev., 138, 3572-3578, https:// doi.org/10.1175/2010MWR3441.1.
_ 2012: Impacts of lapse rates on low-level rotation in idealized storms. J. Atmos. Sci., 69, 538-559, https://doi.org/10.1175/ JAS-D-11-058.1.

_ 2017: How much does "backing aloft" actually impact a supercell? Wea. Forecasting, 32, 1937-1957, https://doi.org/ 10.1175/WAF-D-17-0064.1.

— , and R. H. Johnson, 2004a: Simulated convective lines with leading precipitation. Part I: Governing dynamics. J. Atmos. Sci., 61, 1637-1655, https://doi.org/10.1175/1520-0469(2004) 061<1637:SCLWLP>2.0.CO;2.

, and $-2004 \mathrm{~b}$ : Structures and dynamics of quasi-2D mesoscale convective systems. J. Atmos. Sci., 61, 545-567, https:// doi.org/10.1175/1520-0469(2004)061<0545:SADOQM > 2.0.CO;2.

Przybylinski, R. W., G. K. Schmocker, and Y.-J. Lin, 2000: A study of storm and vortex morphology during the "intensifying stage" of severe wind mesoscale convective systems. Preprints, 20th Conf. on Severe Local Storms, Orlando, FL, Amer. Meteor. Soc., 173-176.

Roberts, B., M. Xue, A. D. Schenkman, and D. T. Dawson II, 2016: The role of surface drag in tornadogenesis within an idealized supercell simulation. J. Atmos. Sci., 73, 3371-3395, https:// doi.org/10.1175/JAS-D-15-0332.1.

Rotunno, R., and J. B. Klemp, 1982: The influence of the shearinduced pressure gradient on thunderstorm motion. Mon. Wea. Rev., 110, 136-151, https://doi.org/10.1175/1520-0493(1982) $110<0136$ :TIOTSI $>2.0$. CO;2.

— P. M. Markowski, and G. H. Bryan, 2017: "Near ground" vertical vorticity in supercell thunderstorm models. J. Atmos. Sci., 74, 1757-1766, https://doi.org/10.1175/JAS-D-16-0288.1.

Schaumann, J. S., and R. W. Przybylinski, 2012: Operational application of $0-3 \mathrm{~km}$ bulk shear vectors in assessing quasi linear convective system mesovortex and tornado potential. 26th Conf. on Severe Local Storms, Nashville, TN, Amer. Meteor. Soc., 142, https://ams.confex.com/ams/26SLS/webprogram/ Paper212008.html.

Schenkman, A. D., M. Xue, and A. Shapiro, 2012: Tornadogenesis in a simulated mesovortex within a mesoscale convective system. J. Atmos. Sci., 69, 3372-3390, https://doi.org/10.1175/ JAS-D-12-038.1.

,-- , and M. Hu, 2014: Tornadogenesis in a high-resolution simulation of the 8 May 2003 Oklahoma City supercell. J. Atmos. Sci., 71, 130-154, https://doi.org/10.1175/JAS-D13-073.1.

Schneider, R. S., A. R. Dean, S. J. Weiss, and P. D. Bothwell, 2006: Analysis of estimated environments for 2004 and 2005 severe convective storm reports. 23rd Conf. on Severe Local Storms, St. Louis, MO, Amer. Meteor. Soc., 3.5, https://ams.confex.com/ ams/23SLS/techprogram/paper_115246.htm.

Sherburn, K. D., and M. D. Parker, 2014: Climatology and ingredients of significant severe convection in high-shear, lowCAPE environments. Wea. Forecasting, 29, 854-877, https:// doi.org/10.1175/WAF-D-13-00041.1.

_ _ _ J. R. King, and G. M. Lackmann, 2016: Composite environments of severe and nonsevere high-shear, low-CAPE convective events. Wea. Forecasting, 31, 1899-1927, https:// doi.org/10.1175/WAF-D-16-0086.1.

, and Coauthors, 2019: Partnering research, education, and operations via a cool season severe weather soundings program. Bull. Amer. Meteor. Soc., 100, 307-320, https://doi.org/ 10.1175/BAMS-D-17-0186.1.

Smith, B. T., R. L. Thompson, J. S. Grams, C. Broyles, and H. E. Brooks, 2012: Convective modes for significant severe 
thunderstorms in the contiguous United States. Part I: Storm classification and climatology. Wea. Forecasting, 27, 11141135, https://doi.org/10.1175/WAF-D-11-00115.1.

Trapp, R. J., and M. L. Weisman, 2003: Low-level mesovortices within squall lines and bow echoes. Part II: Their genesis and implications. Mon. Wea. Rev., 131, 2804-2823, https://doi.org/ 10.1175/1520-0493(2003)131<2804:LMWSLA > 2.0.CO;2.

Wakimoto, R. M., H. V. Murphey, A. Nester, D. P. Jorgensen, and N. T. Atkins, 2006: High winds generated by bow echoes. Part I: Overview of the Omaha bow echo 5 July 2003 storm during BAMEX. Mon. Wea. Rev., 134, 2793-2812, https://doi.org/ 10.1175/MWR3215.1.

Weisman, M. L., and J. B. Klemp, 1984: The structure and classification of numerically simulated convective storms in directionally varying wind shears. Mon. Wea. Rev., 112, 2479-2498, https:// doi.org/10.1175/1520-0493(1984)112<2479:TSACON>2.0.CO;2. , and R. Rotunno, 2000: The use of vertical wind shear versus helicity in interpreting supercell dynamics. J. Atmos. Sci., 57,
1452-1472, https://doi.org/10.1175/1520-0469(2000)057<1452: TUOVWS $>2.0 . \mathrm{CO} ; 2$.

—_, and R. J. Trapp, 2003: Low-level mesovortices within squall lines and bow echoes. Part I: Overview and dependence on environmental shear. Mon. Wea. Rev., 131, 2779-2803, https:// doi.org/10.1175/1520-0493(2003)131<2779:LMWSLA > 2.0.CO;2.

Weiss, J., 1991: The dynamics of enstrophy transfer in twodimensional hydrodynamics. Physica D, 48, 273-294, https:// doi.org/10.1016/0167-2789(91)90088-Q.

Wilhelmson, R. B., and Y. Ogura, 1972: The pressure perturbation and the numerical modeling of a cloud. J. Atmos. Sci., 29 , 1295-1307, https://doi.org/10.1175/1520-0469(1972)029<1295: TPPATN $>2.0 . \mathrm{CO} ; 2$.

Xu, X., M. Xue, and Y. Wang, 2015: The genesis of mesovortices within a real-data simulation of a bow echo system. J. Atmos. Sci., 72, 1963-1986, https://doi.org/10.1175/JASD-14-0209.1. 


\section{Corrigendum}

\section{Keith D. Sherburn ${ }^{\mathrm{a}}$ AND MATTHEw D. PARKeR ${ }^{\mathrm{a}}$}

${ }^{a}$ Department of Marine, Earth, and Atmospheric Sciences, North Carolina State University, Raleigh, North Carolina

(Manuscript received 17 December 2020, in final form 24 February 2021)

Through another research effort, it was recently discovered that the wind profiles used to initialize the Cloud Model 1 (CM1) simulations in Sherburn and Parker (2019) were specified with incorrect units (mistaking meters per second for knots). The following corrected versions of Fig. 1 and Table 1 represent the actual initial conditions for the simulations reported in Sherburn and Parker (2019).

The caption for Fig. 3 in Sherburn and Parker (2019) should also be modified to read, "Base-state kinematic profiles for the (left) increased low-level shear and (right) decreased low-level shear simulations. Hodograph axes are labeled in $\mathrm{m} \mathrm{s}^{-1}$."

The modeling results shown and interpreted by Sherburn and Parker (2019) remain true to the CM1 simulations that they actually performed. These results are internally consistent with one another and do indeed address the modeled convection's sensitivities to changes in vertical wind shear and stability, which was the study's purpose. However, the initial environments in the model contained approximately twice as much vertical shear as intended.

\section{REFERENCE}

Sherburn, K. D., and M. D. Parker, 2019: The development of severe vortices within simulated high-shear low-CAPE convection. Mon. Wea. Rev., 147, 2189-2216, https://doi.org/10.1175/MWR-D-18-0246.1.

Sherburn's current affiliation: National Weather Service Forecast Office, Rapid City, South Dakota.

Corresponding author: Keith D. Sherburn, keith.sherburn@noaa.gov 


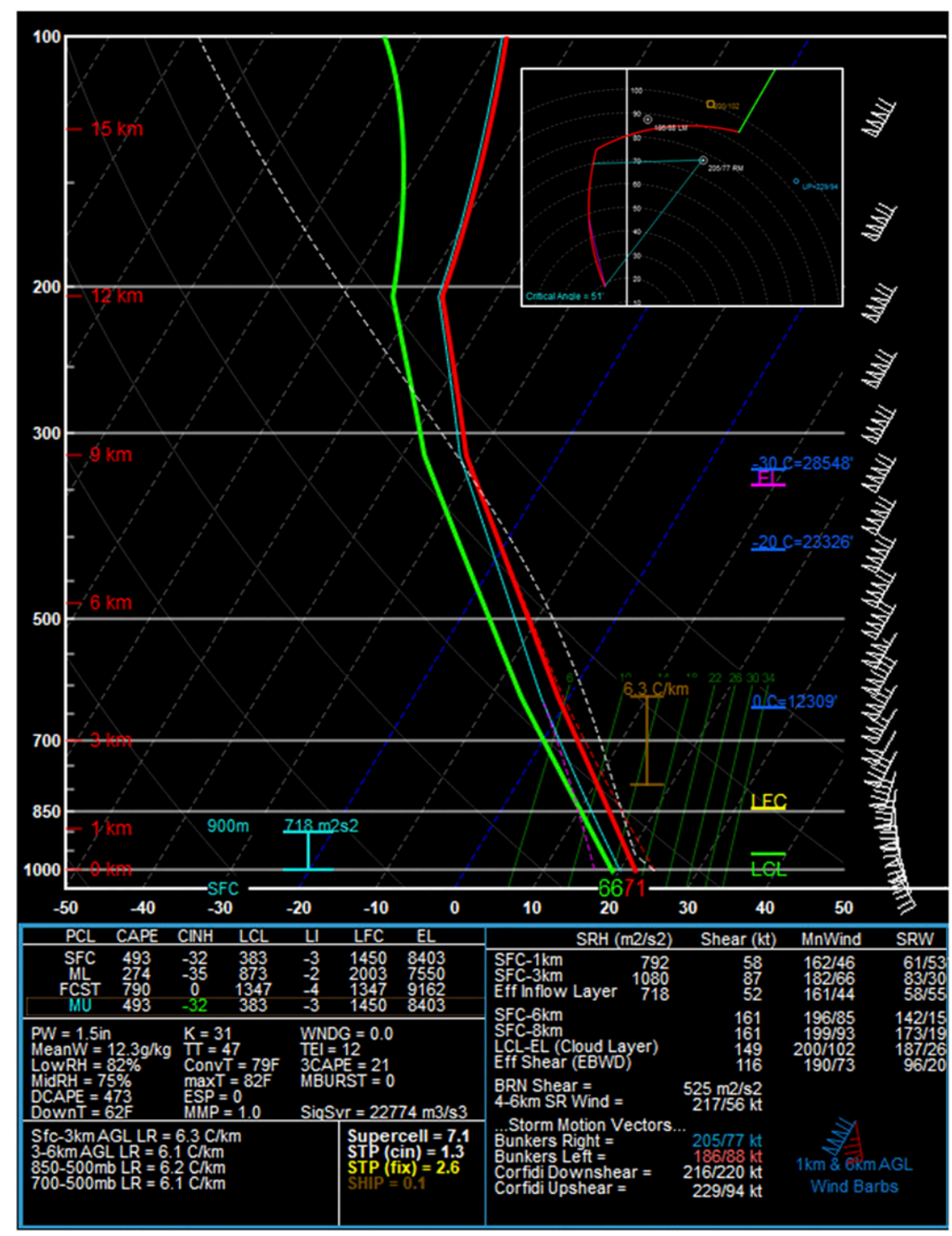

FIG. 1. Control base-state environment in HSLC matrix of simulations. Hodograph axes are labeled in knots $\left(1 \mathrm{kt}=0.51444 \mathrm{~m} \mathrm{~s}^{-1}\right)$ and contoured at $10-\mathrm{kt}$ intervals. Half barbs, barbs, and flags on the right correspond to wind magnitudes of 5,10 , and $50 \mathrm{kt}$, respectively. 
TABLE 1. Selected base-state environment variables for matrix of simulations.

\begin{tabular}{|c|c|c|c|c|c|}
\hline Variable & Control & $+01 \mathrm{~s}$ & $-01 \mathrm{~s}$ & $+031 \mathrm{r}$ & $-031 \mathrm{r}$ \\
\hline SBCAPE $\left(\mathrm{J} \mathrm{kg}^{-1}\right)$ & 493 & 493 & 493 & 493 & 493 \\
\hline $\operatorname{MLCAPE}\left(\mathrm{J} \mathrm{kg}^{-1}\right)$ & 274 & 274 & 274 & 276 & 288 \\
\hline $0-3-\mathrm{km} \mathrm{CAPE}\left(\mathrm{J} \mathrm{kg}^{-1}\right)$ & 21 & 21 & 21 & 40 & 6 \\
\hline $0-1-\mathrm{km}$ shear $\left(\mathrm{m} \mathrm{s}^{-1}\right)$ & 30 & 40 & 20 & 30 & 30 \\
\hline $0-1-\mathrm{km}$ SRH $\left(\mathrm{m}^{2} \mathrm{~s}^{-2}\right)$ & 792 & 1121 & 491 & 729 & 834 \\
\hline $0-3-\mathrm{km}$ shear $\left(\mathrm{m} \mathrm{s}^{-1}\right)$ & 45 & 45 & 45 & 45 & 45 \\
\hline $0-3-\mathrm{km}$ SRH $\left(\mathrm{m}^{2} \mathrm{~s}^{-2}\right)$ & 1080 & 1390 & 836 & 1067 & 1084 \\
\hline $0-6-\mathrm{km}$ shear $\left(\mathrm{m} \mathrm{s}^{-1}\right)$ & 83 & 83 & 83 & 83 & 83 \\
\hline
\end{tabular}

